\title{
High-Throughput Single-Molecule Spectroscopy Resolves the Conformational Isomers of
}

\section{BODIPY Chromophores}

Lorenzo Sansalone, $\uparrow$ Yang Zhang, $\uparrow, \S, *$ Mercedes M. A. Mazza, $\uparrow$ Ki-Hee Song,§ Janel L. Davis,§ Burjor Captain, $\uparrow$ Hao F. Zhang§ and Françisco M. Raymo*,†

$\dagger$ Laboratory for Molecular Photonics, Department of Chemistry, University of Miami, 1301 Memorial Drive, Coral Gables, FL 33146-0431

$\S$ Departments of Biomedical Engineering, Northwestern University, 2145 Sheridan Road, Evanston, IL 60208

- Procedures

- Crystallographic Data.

- Computational Data .S8

- Spectroscopic Data. .$S 32$

* Corresponding Authors yang.zhangfl@gmail.com fraymo@miami.edu 


\section{Experimental Procedures}

Materials and Methods. Chemicals were purchased from commercial sources and used as received. $\mathrm{CH}_{2} \mathrm{Cl}_{2}$ and $\mathrm{MeCN}_{\text {were }}$ distilled over $\mathrm{CaH}_{2}$. Tetrahydrofuran (THF) was distilled over $\mathrm{Na}$ and benzophenone. $\mathrm{H}_{2} \mathrm{O}(18.2 \mathrm{M} \Omega \mathrm{cm})$ was purified with a Barnstead International NANOpure Diamond analytical system. Compounds 3, 4 and $\mathbf{8}$ were prepared according to a literature procedure.s 1,S2 Electrospray ionization mass spectra (ESIMS) were recorded with a Bruker micrOTO-Q II spectrometer. Nuclear magnetic resonance (NMR) spectra were recorded with Bruker 300, 400 and 500 spectrometers. Absorption spectra were recorded with a Varian Cary 100 Bio spectrometer in quartz cells with path length of $1.0 \mathrm{~cm}$. Emission and excitation spectra were recorded with a Varian Cary Eclipse spectrometer in aerated solutions. Fluorescence quantum yields were determined against a solution of $\mathbf{8}(\phi=0.50) \mathrm{s} 2$ in $\mathrm{MeCN}$, following a literature protocol.s3

1. $N$-Bromosuccinimide (NBS, $1.34 \mathrm{~g}, 7.5 \mathrm{mmol}$ ) and azobis-iso-butyrronitrile (AIBN, $62.4 \mathrm{mg}, 0.4 \mathrm{mmol}$ ) were added to a solution of 2-methylbenzoxazole $(1.00 \mathrm{~g}, 7.5 \mathrm{mmol})$ in $\mathrm{CCl}_{4}(10 \mathrm{~mL})$ stirred at ambient temperature under Ar. The reaction mixture was warmed up to $70{ }^{\circ} \mathrm{C}$ and stirred at this temperature for 19 hours. The resulting suspension was cooled down to ambient temperature and filtered. The solvent of the filtrate was distilled off under reduced pressure and the residue was purified by column chromatography [ $\mathrm{SiO}_{2}$, hexane/ $\left.\mathrm{CH}_{2} \mathrm{Cl}_{2} / \mathrm{EtOAc}(30: 10: 1, \mathrm{v} / \mathrm{v} / \mathrm{v})\right]$ to afford $1(0.65 \mathrm{~g}, 41 \%)$. ESIMS: $\mathrm{m} / \mathrm{z}$ calcd for $\mathrm{C}_{8} \mathrm{H}_{6} \mathrm{BrNNaO}_{+}=234.0 ; \mathrm{m} / z$ found $=234.0[\mathrm{M}+\mathrm{Na}]_{+} ;{ }_{1} \mathrm{H} \mathrm{NMR}\left(300 \mathrm{MHz}, \mathrm{CDCl}_{3}\right): \delta=7.77-7.74(\mathrm{~m}, 1 \mathrm{H}), 7.60$ $7.54(\mathrm{~m}, 1 \mathrm{H}), 7.45-7.35(\mathrm{~m}, 2 \mathrm{H}), 4.62(\mathrm{~s}, 2 \mathrm{H})$.

2. A suspension of $\mathbf{1}(0.63 \mathrm{~g}, 3.0 \mathrm{mmol})$ in $\mathrm{P}(\mathrm{OEt}) 3(1.02 \mathrm{~mL}, 5.98 \mathrm{mmol})$ was heated under reflux for 2 hours. After cooling down to ambient temperature, the mixture was diluted with EtOAc $(5 \mathrm{~mL})$ and washed with aqueous $\mathrm{HCl}(1 \mathrm{M}, 3 \times 10 \mathrm{~mL})$. The organic phase was dried over $\mathrm{Na}_{2} \mathrm{SO}_{4}$ and the solved was distilled off under reduced pressure. The residue was purified by column chromatography $\left[\mathrm{SiO}_{2}, \mathrm{CH}_{2} \mathrm{Cl}_{2} / \mathrm{EtOAc}(50: 50, \mathrm{v} / \mathrm{v})\right]$ to afford $2(0.29 \mathrm{~g}, 36 \%)$ as clear liquid. ESIMS: $\mathrm{m} / \mathrm{z}$ calcd for $\mathrm{C}_{12} \mathrm{H}_{16} \mathrm{NO}_{4} \mathrm{P}=269.0817 ; \mathrm{m} / z$ found $=270.0899[\mathrm{M}+\mathrm{H}]+;{ }_{1} \mathrm{H}$ NMR $\left(300 \mathrm{MHz}, \mathrm{CDCl}_{3}\right): \delta=7.73-7.69(\mathrm{~m}, 1 \mathrm{H}), 7.58-$ $7.52(\mathrm{~m}, 1 \mathrm{H}), 7.39-7.32(\mathrm{~m}, 2 \mathrm{H}), 4.27-4.16(\mathrm{~m}, 4 \mathrm{H}), 3.63(\mathrm{~s}, 1 \mathrm{H}), 3.56(\mathrm{~s}, 1 \mathrm{H}), 1.35(\mathrm{~m}, 6 \mathrm{H})$.

BO. A solution of $n$-Buli $(0.41 \mathrm{mmol}, 2.5 \mathrm{M})$ in hexane $(0.16 \mathrm{~mL})$ was added dropwise to a solution of $2(100 \mathrm{mg}, 0.37$ $\mathrm{mmol})$ in THF $(5 \mathrm{~mL})$ stirred at $-78{ }^{\circ} \mathrm{C}$ under Ar. After 15 minutes of stirring at this temperature, a solution of $\mathbf{3}(0.15 \mathrm{~g}$, $0.37 \mathrm{mmol})$ in THF $(3 \mathrm{~mL})$ was added dropwise and the mixture was allowed to warm up to ambient temperature with stirring and maintained under these conditions for a further 15 hours. The solvent was distilled off under reduced pressure and the residue was dissolved in EtOAc $(15 \mathrm{~mL})$ and washed with a saturated aqueous solution of $\mathrm{NaCl}(3 \times 20 \mathrm{~mL})$. The organic phase was dried over $\mathrm{Na}_{2} \mathrm{SO}_{4}$ and the solved was distilled off under reduced pressure. The residue was purified by column chromatography $\left[\mathrm{SiO}_{2}\right.$, hexane/ $\mathrm{CH}_{2} \mathrm{Cl}_{2} / \mathrm{EtOAc}(80: 15: 5, \mathrm{v} / \mathrm{v}) \rightarrow \mathrm{CHCl}_{3} /$ hexane $\left.(50: 10, \mathrm{v} / \mathrm{v})\right]$ to afford $\mathbf{B O}(40 \mathrm{mg}, 21 \%)$ as a dark gold solid. ESIMS: $\mathrm{m} / z$ calcd for $\mathrm{C}_{31} \mathrm{H}_{30} \mathrm{BF}_{2} \mathrm{~N}_{3} \mathrm{O}=509.2450 ; \mathrm{m} / z$ found $=510.2529[\mathrm{M}+\mathrm{H}]_{+} ;{ }_{1} \mathrm{H} \mathrm{NMR}(300 \mathrm{MHz}$,

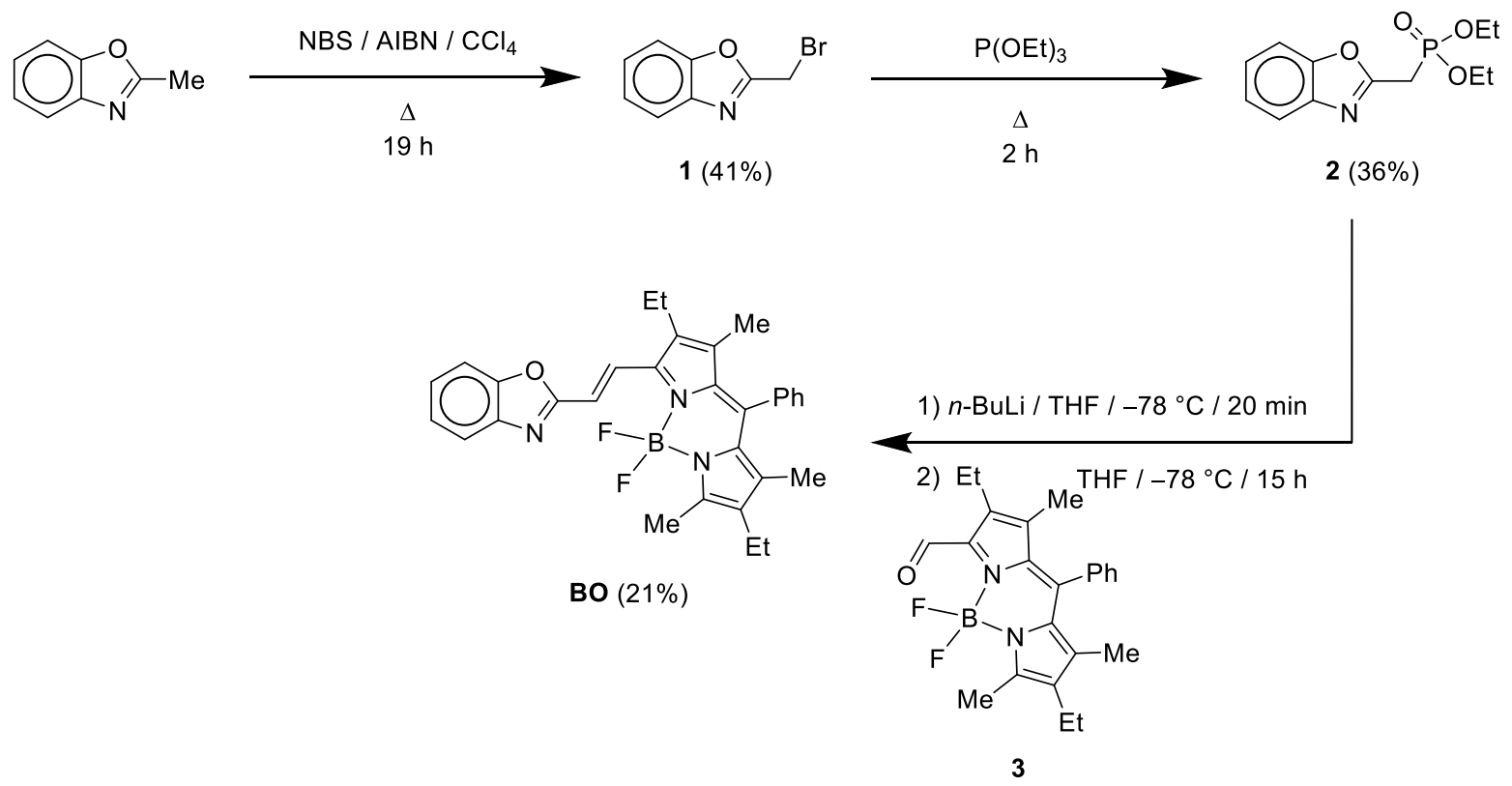

Figure S1. Synthesis of BO. 


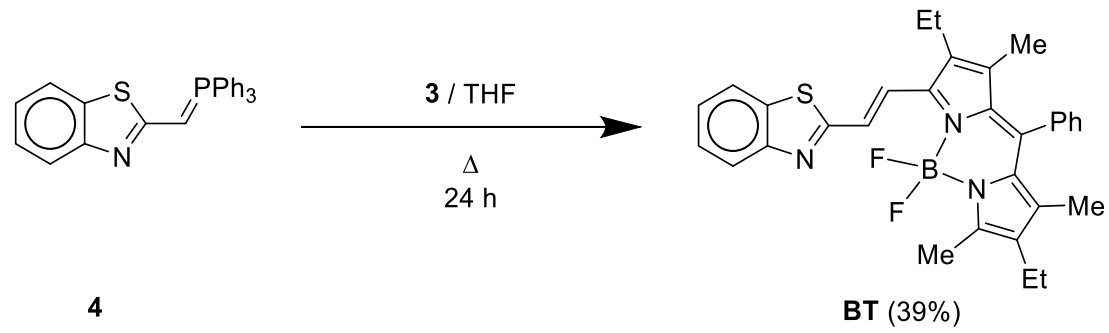

Figure S2. Synthesis of BT.

$\left.\mathrm{CDCl}_{3}\right): \delta=8.29(\mathrm{~d}, 16.7 \mathrm{~Hz}, 1 \mathrm{H}), 7.78-7.66(\mathrm{~m}, 1 \mathrm{H}), 7.60(\mathrm{dd}, 6.8$ and $2.3 \mathrm{~Hz}, 1 \mathrm{H}), 7.57-7.46(\mathrm{~m}, 3 \mathrm{H}), 7.44-7.29(\mathrm{~m}, 4 \mathrm{H})$, $7.17(\mathrm{~d}, 16.7 \mathrm{~Hz}, 1 \mathrm{H}), 2.77-2.54(\mathrm{~m}, 5 \mathrm{H}), 2.35$ (q, $7.5 \mathrm{~Hz}, 2 \mathrm{H}), 1.33(\mathrm{~m}, 6 \mathrm{H}), 1.17$ (t, $7.5 \mathrm{~Hz}, 3 \mathrm{H}), 1.02(\mathrm{t}, 7.5 \mathrm{~Hz}, 3 \mathrm{H})$.

BT. A solution of $3(50 \mathrm{mg}, 0.13 \mathrm{mmol})$ and $4(121 \mathrm{mg}, 0.29 \mathrm{mmol})$ in THF (25 mL) was stirred under reflux and Ar for 24 hours. After cooling down to ambient temperature, the solvent was distilled off under reduced pressure and the residue was purified by column chromatography $\left[\mathrm{SiO}_{2}, \mathrm{CH}_{2} \mathrm{Cl}_{2}\right]$ to afford BT $(27 \mathrm{mg}, 39 \%)$ as a gold solid. ESIMS: $\mathrm{m} / z$ calcd for $\mathrm{C}_{31} \mathrm{H}_{30} \mathrm{BF}_{2} \mathrm{~N}_{3} \mathrm{~S}=525.2222 ; \mathrm{m} / z$ found $=526.2302[\mathrm{M}+\mathrm{H}]_{+} ;{ }_{1} \mathrm{H} \mathrm{NMR}\left(400 \mathrm{MHz}, \mathrm{CDCl}_{3}\right): \delta=8.07(\mathrm{~d}, 16.8 \mathrm{~Hz}, 1 \mathrm{H}), 8.01(\mathrm{~d}$, $8.1 \mathrm{~Hz}, 1 \mathrm{H}), 7.87(\mathrm{~d}, 7.9 \mathrm{~Hz}, 1 \mathrm{H}), 7.60-7.43(\mathrm{~m}, 5 \mathrm{H}), 7.43-7.29(\mathrm{~m}, 3 \mathrm{H}), 2.74-2.56(\mathrm{~m}, 5 \mathrm{H}), 2.35$ (q, $7.7 \mathrm{~Hz}, 2 \mathrm{H}), 1.39-1.29$ $(\mathrm{m}, 6 \mathrm{H}), 1.17(\mathrm{t}, 7.6 \mathrm{~Hz}, 3 \mathrm{H}), 1.02(\mathrm{t}, 7.6 \mathrm{~Hz}, 3 \mathrm{H})$.

5. NBS (1.83 g, $10.31 \mathrm{mmol})$ and AIBN (169 mg, $1.03 \mathrm{mmol})$ were added to a solution of 2-methyl-5-nitrobenzothiazole (2 $\mathrm{g}, 10.31 \mathrm{mmol})$ in $\mathrm{CCl}_{4}(20 \mathrm{~mL})$ stirred at ambient temperature under Ar. The reaction mixture was warmed up to $70{ }^{\circ} \mathrm{C}$ and stirred at this temperature for 5 hours. The resulting suspension was cooled down to ambient temperature and filtered. The solvent of the filtrate was distilled off under reduced pressure and the residue was purified by column chromatography $\left[\mathrm{SiO}_{2}\right.$, hexane/ EtOAc (60:10 v/v)] to afford $5(0.42 \mathrm{~g}, 15 \%)$. ${ }_{1} \mathrm{H} \mathrm{NMR}\left(300 \mathrm{MHz}, \mathrm{CDCl}_{3}\right) \delta=8.85(\mathrm{~d}, 2.2 \mathrm{~Hz}, 1 \mathrm{H}), 8.49-8.32(\mathrm{~m}$, $1 \mathrm{H}), 8.14(\mathrm{dd}, 9.1$ and $1.2 \mathrm{~Hz}, 1 \mathrm{H}), 4.86(\mathrm{~s}, 2 \mathrm{H})$.

6. A solution of $5(0.38 \mathrm{~g}, 1.40 \mathrm{mmol})$ and $\mathrm{PPh}_{3}(0.47 \mathrm{~g}, 1.68 \mathrm{mmol})$ in $\mathrm{PhMe}(7 \mathrm{~mL})$ was heated under reflux for 24 hours. After cooling down to ambient temperature, the mixture was filtered and the solid residue was dried under vacuum to afford $6(0.41 \mathrm{~g}, 60 \%)$ as a sticky solid. $1 \mathrm{H}$ NMR $\left(300 \mathrm{MHz}, \mathrm{DMSO}-d_{6}\right) \delta=9.19-9.11(\mathrm{~m}, 1 \mathrm{H}), 8.34-7.48(\mathrm{~m}, 1 \mathrm{H}), 8.06-7.99(\mathrm{~m}$, $1 \mathrm{H}), 7.95-7.48(\mathrm{~m}, 15 \mathrm{H}), 6.17(\mathrm{~d}, 15.8 \mathrm{~Hz}, 2 \mathrm{H})$.

7. $t$-BuOK (130 mg, $1.16 \mathrm{mmol})$ was added to a solution of $6(400 \mathrm{mg}, 0.75 \mathrm{mmol})$ in PhMe $(5 \mathrm{~mL})$ stirred at ambient temperature under Ar. The mixture was stirred under these conditions for 14 hours and filtered. The solvent of the filtrate was distilled off under reduced pressure and the residue was dissolved in the minimum amount of $\mathrm{CH}_{2} \mathrm{Cl}_{2}$. $\mathrm{Hexane}$ was gradually added to obtain a turbid solution, which was stored in the freezer overnight. The resulting red crystals were filtered
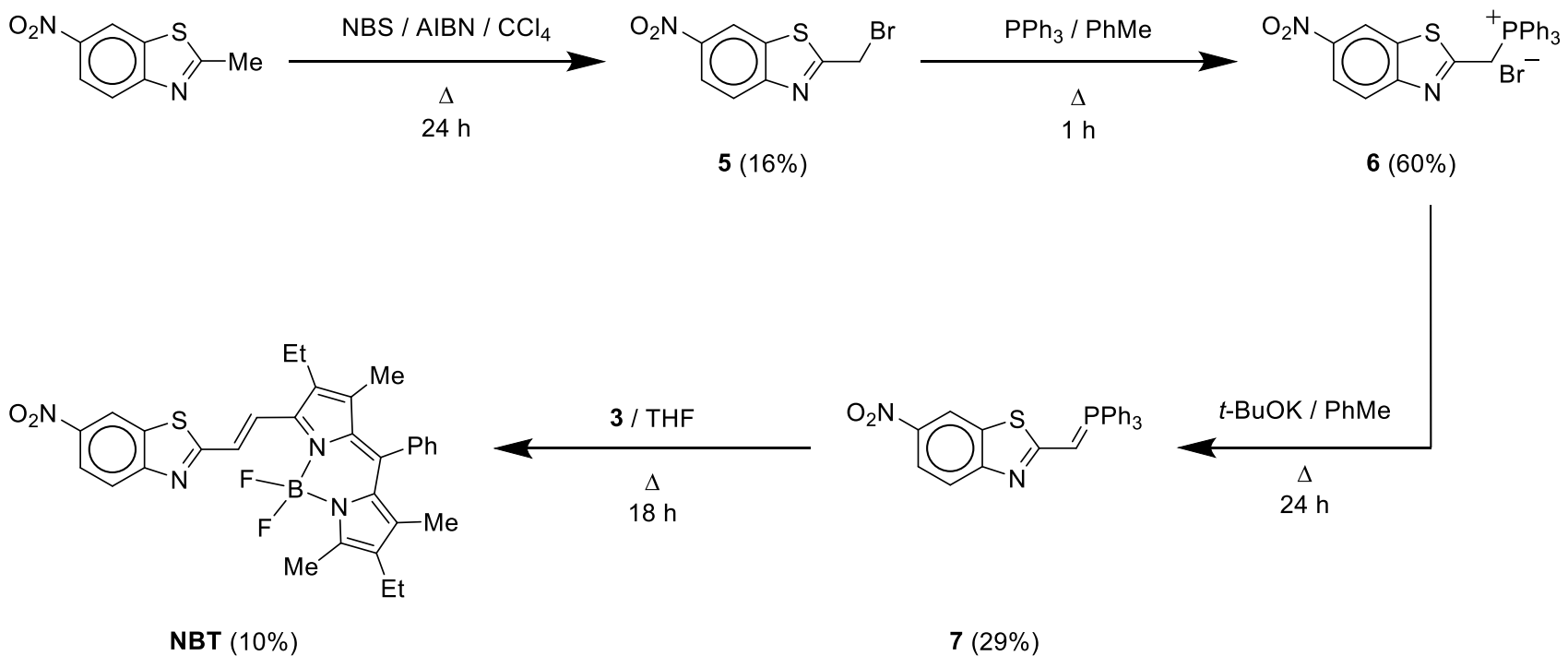

Figure S3. Synthesis of NBT. 
off and dried to afford 7 (100 mg, $29 \%)$. $1 \mathrm{H}$ NMR (300 MHz, DMSO- $\left.d_{6}\right): \delta=8.37(\mathrm{~m}, 1 \mathrm{H}), 7.87(\mathrm{~m}, 1 \mathrm{H}), 7.80-7.51(\mathrm{~m}$, $16 \mathrm{H}), 6.89(\mathrm{~d}, 9.2 \mathrm{~Hz}, 1 \mathrm{H})$.

NBT. A solution of $3(50 \mathrm{mg}, 0.13 \mathrm{mmol})$ and $7(90 \mathrm{mg}, 0.19 \mathrm{mmol})$ in THF $(5 \mathrm{~mL})$ was heated under reflux for 18 hours. After cooling down to ambient temperature, the solvent was distilled off under reduced pressure and the reside was purified by column chromatography $\left[\mathrm{SiO}_{2}\right.$, hexane/EtOAc/ $\left.\mathrm{CH}_{2} \mathrm{Cl}_{2}(80: 10: 10, \mathrm{v} / \mathrm{v} / \mathrm{v})\right]$ to afford $\mathbf{N B T}(7.5 \mathrm{mg}, 10 \%)$ as a yellow solid. ESIMS: $m / z$ calcd for $\mathrm{C}_{31} \mathrm{H}_{29} \mathrm{BF}_{2} \mathrm{~N}_{4} \mathrm{O}_{2} \mathrm{~S}=570.2072 ; m / z$ found: $571.2140[\mathrm{M}+\mathrm{H}]_{+} ; 1 \mathrm{H}$ NMR $\left(500 \mathrm{MHz}, \mathrm{CDCl}_{3}\right) \delta=8.79(\mathrm{~d}$, $2.4 \mathrm{~Hz}, 1 \mathrm{H}), 8.36(\mathrm{dd}, 9.0$ and $2.3 \mathrm{~Hz}, 1 \mathrm{H}), 8.18(\mathrm{~d}, 16.7 \mathrm{~Hz}, 1 \mathrm{H}), 8.07(\mathrm{~d}, 9.0 \mathrm{~Hz}, 1 \mathrm{H}), 7.73-7.65(\mathrm{~m}, 1 \mathrm{H}), 7.56-7.52(\mathrm{~m}$, 3H), 7.36-7.30 (m, 2H), 2.72-2.60 (m, 5H), 2.37 (q, $7.4 \mathrm{~Hz}, 2 \mathrm{H}), 1.35(\mathrm{~s}, 3 \mathrm{H}), 1.34(\mathrm{~s}, 3 \mathrm{H}), 1.18$ (t, $7.5 \mathrm{~Hz}, 3 \mathrm{H}), 1.04$ (t, 7.5 $\mathrm{Hz}, 3 \mathrm{H})$.

Crystallographic Analysis. Single crystals of BT, suitable for diffraction analysis, grew from a $\mathrm{CH}_{2} \mathrm{Cl}_{2}$ solution of the compound maintained at ambient temperature, after diffusion of pentane vapors. The data crystal was mounted onto the end of a thin glass fiber using Paratone-N for data collection at $100 \mathrm{~K}$ under $\mathrm{N}_{2}$. X-ray intensity data were measured by using a Bruker SMART APEX2 CCD-based diffractometer using Mo K $\alpha$ radiation $(\lambda=0.71073 \AA$ ).s4 The raw data frames were integrated with the SAINT+ program by using a narrow-frame integration algorithm.s4 Corrections for Lorentz and polarization effects were also applied with SAINT+. An empirical absorption correction based on the multiple measurement of equivalent reflections was applied using the program SADABS. The structure was solved by a combination of direct methods and difference Fourier syntheses and refined by full-matrix least-squares on F2, using the SHELXTL software package. $55, \mathrm{~s} 6$ All non-hydrogen atoms were refined with anisotropic displacement parameters. Hydrogen atoms were placed in geometrically-idealized positions and included, as standard riding atoms, during the least-squares refinements. Crystal data, data collection parameters and results of the analyses are listed in Table S1. Compound BT crystallized in the monoclinic crystal system. The systematic absences in the intensity data identified the unique space group $P 21 / n$.

Computational Methods. Density-functional theorys7 (DFT) calculations were performed with the 6-311+G(d,p) basis set and the restricted B3LYPs8,s9 and M06HFs10,s11 functionals implemented in Gaussian 09.s12 Geometry optimizations and frequency calculations were carried out with the polarizable continuum model (PCM) for acetonitrile, using the integral equation formalism (IEF) variant.s13 Molecular orbitals were computed at the same level of theory, including the solvation model. Single-point second-order Møller-Plesset perturbation (MP2) theorys 14 calculations were performed on the optimized geometries with the same basis set and solvation model. Time-dependents15 (TD) DFT calculations were performed with the same basis set, functionals and solvation model of the ground-state calculations.

Single-Molecule Spectroscopy. The optical setup for single-molecule imaging and spectroscopy was adapted from a literature precedent.s16 Specifically, a continuous-wave laser was used for excitation (532 nm, $200 \mathrm{~mW}$; Spectra Physics). The laser beam was guided into an inverted microscope (Nikon Ti-E) and subsequently focused by a lens (focal length $=400 \mathrm{~mm}$ ) into the back focal plane of a total internal reflection (TIRF) objective (Nikon CFI Apochromat 100×, numerical aperture $=1.49$ ). The collected fluorescence image was transmitted into a spectrometer, which consists of a transmission-type diffraction grating (100 grooves mm-1, Panton Hawskely Education Ltd. STAR100) and two relay lenses (focal length $=50 \mathrm{~mm}$ ). The image was split into a non-dispersed spatial image and a dispersed first-order spectral image at the grating. The distinguished spatial and spectral images were then captured by an electron-multiplying charge-coupled (EMCCD) camera (Andor iXon 897) simultaneously. A neutral density filter was inserted in the excitation beam line to adjust the beam power. Singlemolecule imaging was performed using TIRF illumination with the excitation intensity of $\sim 200 \mathrm{~W} \mathrm{~cm}-2$ and the exposure time of $30 \mathrm{~ms}$.

The time-dependent measurements for single-molecule spectral trajectory and fluctuation are acquired with the entrance slit width of $5 \mathrm{~mm}$ (wide-slit configuration) and concentrations of $10 \mathrm{pM}$ with only a few sparse molecules in the entire field of view as illustrated in Figure $3 \boldsymbol{a}$. For high-throughput SMS, we increase the concentration of compounds to $1 \mathrm{nM}$. The presence of single molecules is confirmed by the observations of single-step photobleaching as shown in Figure 3, as well as the narrow full width at half maximum of the point spread function of the diffraction-limited spot (less than $310 \mathrm{~nm}$ with our setup). As can be seen in the raw images ( $\boldsymbol{a}$ in Figure S18), there are significant overlapping between different single-molecule spectra using the wide-slit configuration. To achieve optimal spectral precisionsi7 and minimize the possibility to detect overlapped single-molecule spectra, we adjust the entrance slit width to $50 \mu \mathrm{m}$ (narrow-slit configuration, $\boldsymbol{b}$ in Figure S18) for highthroughput SMS. Under this condition, we collected $\sim 10_{4}$ molecules of each compounds within a few minutes by scanning of the microscope stage laterally. With wide-slit setup and low molecular concentration, SMS can also be still achieved with relatively low speed with scanning larger area of the substrates and results are consistent.

Sample Preparation for Single-Molecule Spectroscopy. Toluene solutions of BO, BT and NBT (10 pM for the acquisition of single-molecule spectra over time, $1 \mathrm{nM}$ for high-throughput SMS) were spin-coated on No. 1 cover glasses with a Laurell 
WS-650SZ-23NPPB spin coater operating at 2,000 rpm for $60 \mathrm{~s}$. Prior to spin coating the cover glasses were cleaned with a South Bay Technology PC 2000 Plasma Cleaner. The microscope stage was scanned manually to $c a .10000 \mu \mathrm{m} 2$ of the coated glass slides to ensure enough sampling. The single-molecule spectra were calibrated using their spatial locations as references and the overlapped spectra were removed using Matlab as detailed below.

Calibration Procedure. First, we acquired a calibration image, which is confined by a narrow slit, using a fluorescence calibration source. By integrating the acquired spectral image along the y-axis, we visualized the multiple emission peaks of the calibration source, centered at 487.7, 546.5, 611.6 and $707.0 \mathrm{~nm}$ ( $\boldsymbol{a}$ in Figure S4). Then, we obtained the calibration curve by fitting a relationship of the pixel positions and wavelengths of these emission peaks with a linear polynomial function (b in Figure S4). We used the calibration curve to determine the $\mathrm{nm} /$ pixel ratio and the pixel shifts between the spatial and spectral images and calibrated emission spectra of single molecules by considering their spatial location and the corresponding pixel shifts.s17 Lastly, the obtained spectra were further interpolated to each wavelength unit from 500-700 nm.

(a)

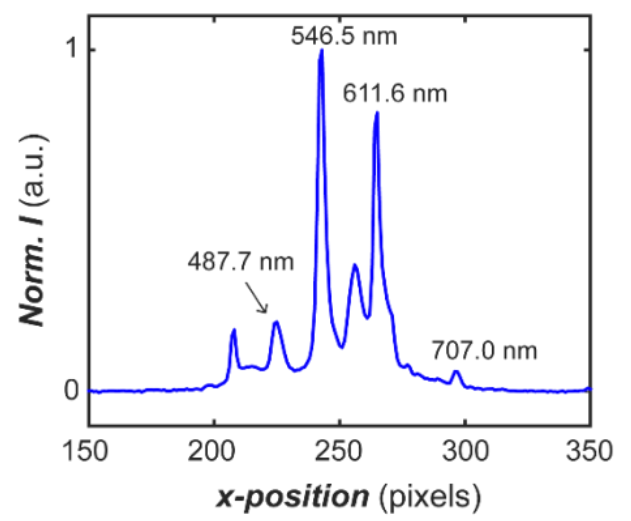

\section{(b)}

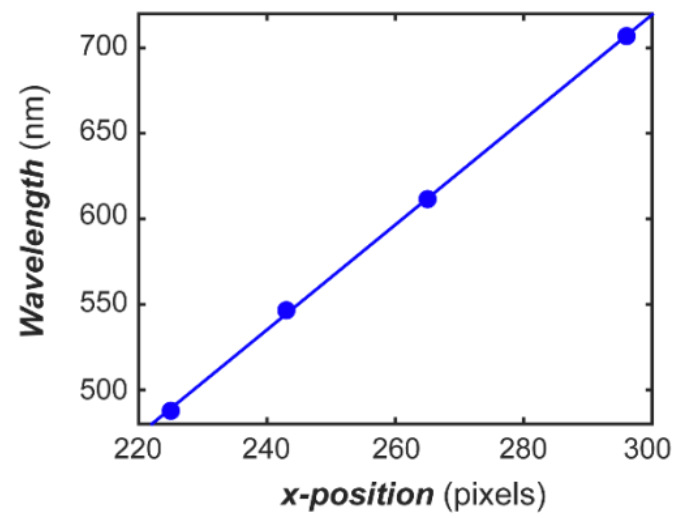

Figure S4. (a) Fluorescence lamp spectrum measured using the single-molecule spectroscopy system. (b) Calibration curve as a function between wavelength and pixel information on the EMCCD camera.

Spectral Centroid Calculation. The spectral centroid (SC) is calculated as the intensity-weighted average wavelength of every single-molecule spectra collecting from a spectral range of 500-700 $\mathrm{nm}$ using the equation below:

$$
\mathrm{SC}=\sum_{n=500}^{n=700} I(n) W(n) / \sum_{n=500}^{n=700} W(n)
$$

where $I(n)$ represents the intensity value at each wavelength after interpolation and $W(n)$ represents the wavelength.

S1 Dondoni, A.; Fantin, G.; Fogagnolo, M.; Medici, A.; Pedrini, P. Thiazolylmethylenetriphenylphosporane and Its Benzo Derivative: Stable and Practical Wittig Reagents for the Synthesis of Vinylthiazoles and Vinylbenzo: Two-Carbon Homologation of Aldehydes. Tetrahedron 1988, 44, $2021-2031$.

S2 Zhang, Y.; Swaminathan, S.; Tang, S.; Garcia-Amorós, J.; Boulina, M.; Captain, B.; Baker, J. D.; Raymo, F. M. Photoactivatable BODIPYs Designed to Monitor the Dynamics of Supramolecular Nanocarriers. J. Am. Chem. Soc. 2015, 137, 4709-4719.

S3 Wurth, C.; Grabolle, M.; Pauli, J.; Spieles, M.; Resch-Genger, U. Relative and Absolute Determination of Fluorescence Quantum Yields of Transparent Samples. Nat. Protocols 2013, 8, 1535-1550.

S4 Apex2 Version 2.2-0 and SAINT+ Version 7.46A; Bruker Analytical X-ray System, Inc.: Madison, Wisconsin, USA, 2007.

S5 Sheldrick, G. M. Crystal Structure Refinement with SHELXL. Acta Crystallogr. C 2015, 71, 3-8.

S6 Sheldrick, G. M. SHELXTL Version 6.1; Bruker Analytical X-ray Systems, Inc., Madison: Wisconsin, USA, 2000.

S7 Parr, R. G.; Yang, W. Density-Functional Theory of Atoms and Molecules; Oxford University Press: Oxford, 1989.

S8 Becke, A. D. Density-Functional Exchange-Energy Approximation with Correct Asymptotic Behavior. Phys. Rev. A 1988, 38, $3098-3100$.

S9 Lee, C.; Yang, W.; Parr, R. G. Development of the Colle-Salvetti Correlation-Energy Formula into a Functional of the Electron Density. Phys. Rev. B 1988, 37, 785-789.

S10 Zhao, Y.; Truhlar, D. G. Density Functional for Spectroscopy: No Long-Range Self-Interaction Error, Good Performance for Rydberg and ChargeTransfer States, and Better Performance on Average than B3LYP for Ground States. J. Phys. Chem. A 2006, 110, 13126-13130. 
S11 Zhao, Y.; Truhlar, D. G. The M06 Suite of Density Functionals for Main Group Thermochemistry, Thermochemical Kinetics, Noncovalent Interactions, Excited States, and Transition Elements: Two New Functionals and Systematic Testing of Four M06-Class Functionals and 12 Other Functionals. Theor. Chem. Acc. 2008, 120, 215-241.

S12 Frisch, M. J.; Trucks, G. W.; Schlegel, H. B.; Scuseria, G. E.; Robb, M. A.; Cheeseman, J. R.; Scalmani, G.; Barone, V.; Mennucci, B.; Petersson, G. A.; Nakatsuji, H.; Caricato, M.; Li, X.; Hratchian, H. P.; Izmaylov, A. F.; Bloino, J.; Zheng, G.; Sonnenberg, J. L.; Hada, M.; Ehara, M.; Toyota, K.; Fukuda, R.; Hasegawa, J.; Ishida, M.; Nakajima, T.; Honda, Y.; Kitao, O.; Nakai, H.; Vreven, T.; Montgomery, J. A. Jr.; Peralta, J. E.; Ogliaro, F.; Bearpark, M.; Heyd, J. J.; Brothers, E.; Kudin, K. N.; Staroverov, V. N.; Keith, T.; Kobayashi, R.; Normand, J.; Raghavachari, K.; Rendell, A.; Burant, J. C.; Iyengar, S. S.; Tomasi, J.; Cossi, M.; Rega, N.; Millam, J. M.; Klene, M.; Knox, J. E.; Cross, J. B.; Bakken, V.; Adamo, C.; Jaramillo, J.; Gomperts, R.; Stratmann, R. E.; Yazyev, O.; Austin, A. J.; Cammi, R.; Pomelli, C.; Ochterski, J. W.; Martin, R. L.; Morokuma, K.; Zakrzewski, V. G.; Voth, G. A.; Salvador, P.; Dannenberg, J. J.; Dapprich, S.; Daniels, A. D.; Farkas, Ö.; Foresman, J. B.; Ortiz, J. V.; Cioslowski, J.; Fox, D. J. Gaussian 09. Revision D.01. Gaussian, Inc., Wallingford CT, 2013. Tomasi, J.; Mennucci, B.; Cammi, R. Quantum Mechanical Continuum Solvation Models. Chem. Rev. 2005, 105, $2999-3093$.

Helgaker, T.; Jorgensen, P.; Olsen, J. Molecular Electronic Structure Theory; Wiley, 2000.

Furche, F.; Ahlrichs, R. Adiabatic Time-Dependent Density Functional Methods for Excited State Properties. J. Chem. Phys. 2002, 117, $7433-7447$.

Zhang, Y.; Song, K.-H.; Dong, B.; Davis, J. L.; Shao, G.; Sun, C.; Zhang, H. F. Multicolor Super-Resolution Imaging Using Spectroscopic SingleMolecule Localization Microscopy with Optimal Spectral Sispersion. Appl. Opt. 2019, 58, 2248-2255.

Song, K.-H.; Dong, B.; Sun, C.; Zhang, H. F. Theoretical Analysis of Spectral Precision in Spectroscopic Single-Molecule Localizatin Microscopy. Rev. Sci. Instrum. 2018, 89, 123703. 
Table S1. Crystallographic Data for BT.

\begin{tabular}{|c|c|}
\hline Empirical Formula & $\mathrm{C}_{31} \mathrm{H}_{30} \mathrm{BF}_{2} \mathrm{~N}_{3} \mathrm{~S}$ \\
\hline Formula Weight & 525.45 \\
\hline Crystal System & Monoclinic \\
\hline \multicolumn{2}{|l|}{ Lattice Parameters } \\
\hline$a(\AA)$ & $10.8426(5)$ \\
\hline$b(\AA)$ & $21.9098(11)$ \\
\hline$c(\AA)$ & $11.8473(6)$ \\
\hline$\beta$ (deg) & $106.849(1)$ \\
\hline$V\left(\AA_{3}\right)$ & $2693.6(2)$ \\
\hline Space Group & $P 2{ }_{1} / n(\# 14)$ \\
\hline Z Value & 4 \\
\hline$\rho_{\text {calc }}\left(\mathrm{g} \mathrm{cm}_{-3}\right)$ & 1.296 \\
\hline$\mu(\mathrm{Mo} \mathrm{K} \alpha)\left(\mathrm{mm}_{-1}\right)$ & 0.160 \\
\hline Temperature (K) & 100 \\
\hline $2 \Theta_{\max }\left({ }^{\circ}\right)$ & 50.00 \\
\hline No. Obs. $[\mathrm{I}>2 \sigma(\mathrm{I})]$ & 3899 \\
\hline No. Parameters & 348 \\
\hline Goodness of Fit & 1.024 \\
\hline Max. Shift in Cycle & 0.003 \\
\hline Residuals*: R1; wR2 & $0.0402 ; 0.0872$ \\
\hline Absorption Correction, & Multi-Scan \\
\hline $\operatorname{Max} / \operatorname{Min}$ & $0.7457 / 0.6500$ \\
\hline Largest Peak in Final Diff. Map (e $\AA_{-3}$ ) & 0.344 \\
\hline
\end{tabular}




\section{Computational Data}

Coordinates of the geometry of isomer ctt of $\mathbf{B T}$ optimized with the B3LYP functional

\begin{tabular}{|c|c|c|c|}
\hline & -4.957389000 & 1.761039000 & 0.22 \\
\hline & -0.252736000 & 1.573714000 & 1.095006000 \\
\hline & -0.335991000 & 1.582222000 & -1.203942000 \\
\hline & -4.973220000 & -0.820595000 & -0.176301000 \\
\hline & 0.433068000 & -0.438485000 & -0.087722000 \\
\hline & 1.827732000 & 1.643775000 & -0.131584000 \\
\hline & 0.383394000 & 1.104213000 & -0.084284000 \\
\hline & -6.504522000 & 0.963854000 & 0.066721000 \\
\hline & -7.789199000 & 1.506825000 & 0.122746000 \\
\hline & -7.942406000 & 2.566815000 & 0.283812000 \\
\hline & -8.870985000 & 0.647086000 & -0.034621000 \\
\hline & -9.877758000 & 1.046106000 & 0.004974000 \\
\hline & -8.676363000 & -0.729709000 & -0.244297000 \\
\hline & -9.537793000 & -1.376380000 & -0.363914000 \\
\hline & -7.400138000 & -1.270410000 & -0.300161000 \\
\hline & -7.243198000 & -2.330405000 & -0.461172000 \\
\hline & -6.291930000 & -0.422000000 & -0.143580000 \\
\hline & -4.158030000 & 0.182206000 & -0.001837000 \\
\hline & -2.711411000 & 0.130062000 & 0.025294000 \\
\hline & -2.201610000 & 1.069899000 & 0.168635000 \\
\hline & -2.032114000 & -1.035005000 & -0.106184000 \\
\hline & -2.641191000 & -1.924852000 & -0.218024000 \\
\hline & -0.620368000 & -1.317202000 & -0.102592000 \\
\hline & -0.110745000 & -2.645535000 & -0.108883000 \\
\hline & -0.936244000 & -3.904498000 & $-0.10^{-}$ \\
\hline & -0.374923000 & -4.700810000 & -0.602826000 \\
\hline & -1.834356000 & -3.762257000 & -0.714998000 \\
\hline & -1.344935000 & -4.375004000 & 1.300326000 \\
\hline & -0.465458000 & -4.571228000 & 1.919435000 \\
\hline & -1.932009000 & -5.295688000 & 1.243030000 \\
\hline ] & -1.948984000 & -3.618997000 & 1.808985000 \\
\hline & 1.281849000 & -2.566486000 & -0.077896000 \\
\hline & 2.200664000 & -3.752836000 & -0.049235000 \\
\hline & 1.627173000 & -4.671536000 & 0.071749000 \\
\hline H & 2.914789000 & -3.696079000 & 0.773305000 \\
\hline & 2.781158000 & -3.841425000 & -0.970673000 \\
\hline & 1.615022000 & -1.177086000 & -0.078859000 \\
\hline & 2.879161000 & -0.535931000 & -0.093156000 \\
\hline & 4.126651000 & -1.359763000 & -0.082492000 \\
\hline & 4.684054000 & -1.815179000 & -1.281418000 \\
\hline & 4.203686000 & -1.574071000 & -2.223071000 \\
\hline & 5.854338000 & -2.572360000 & -1.269623000 \\
\hline & 6.279312000 & -2.918589000 & -2.204904000 \\
\hline & 6.475596000 & -2.883280000 & -0.060112000 \\
\hline & 7.384754000 & -3.473588000 & -0.051399000 \\
\hline & 5.922563000 & -2.431729000 & 1.138118000 \\
\hline & 6.400250000 & -2.669079000 & 2.081878000 \\
\hline & 4.754112000 & -1.671661000 & 1.127691000 \\
\hline & 4.327076000 & -1.320842000 & 2.060513000 \\
\hline & 2.983017000 & 0.852725000 & -0.124569000 \\
\hline & 4.116616000 & 1.740661000 & -0.172332000 \\
\hline & 5.572750000 & 1.389674000 & -0.184048000 \\
\hline
\end{tabular}




$\begin{array}{lccc}\mathrm{H} & 6.177833000 & 2.289496000 & -0.296929000 \\ \mathrm{H} & 5.821245000 & 0.708955000 & -1.000506000 \\ \mathrm{H} & 5.875827000 & 0.897547000 & 0.743316000 \\ \mathrm{C} & 3.609432000 & 3.035740000 & -0.210803000 \\ \mathrm{C} & 4.381379000 & 4.326378000 & -0.256445000 \\ \mathrm{H} & 3.785783000 & 5.086177000 & -0.770464000 \\ \mathrm{H} & 5.280560000 & 4.191982000 & -0.864336000 \\ \mathrm{C} & 4.784823000 & 4.853250000 & 1.133222000 \\ \mathrm{H} & 3.905775000 & 5.035145000 & 1.757551000 \\ \mathrm{H} & 5.336622000 & 5.793046000 & 1.044095000 \\ \mathrm{H} & 5.421342000 & 4.134201000 & 1.655571000 \\ \mathrm{C} & 2.191997000 & 2.938910000 & -0.180350000 \\ \mathrm{C} & 1.219870000 & 4.069407000 & -0.230486000 \\ \mathrm{H} & 0.264574000 & 3.800455000 & 0.215139000 \\ \mathrm{H} & 1.035979000 & 4.365101000 & -1.269546000 \\ \mathrm{H} & 1.621243000 & 4.940293000 & 0.289670000\end{array}$

Coordinates of the geometry of isomer ctc of $\mathbf{B T}$ optimized with the B3LYP functional

$\begin{array}{cccc}\mathrm{S} & -5.201209000 & -1.331006000 & -0.146868000 \\ \mathrm{~F} & -0.306944000 & 1.547662000 & 1.086569000 \\ \mathrm{~F} & -0.362455000 & 1.569939000 & -1.213496000 \\ \mathrm{~N} & -4.810806000 & 1.248496000 & 0.044826000 \\ \mathrm{~N} & 0.443246000 & -0.438067000 & -0.098505000 \\ \mathrm{~N} & 1.785434000 & 1.679435000 & -0.116061000 \\ \mathrm{~B} & 0.354471000 & 1.103272000 & -0.086864000 \\ \mathrm{C} & -6.602874000 & -0.285124000 & -0.074345000 \\ \mathrm{C} & -7.955767000 & -0.623969000 & -0.102374000 \\ \mathrm{H} & -8.274426000 & -1.656283000 & -0.178288000 \\ \mathrm{C} & -8.889321000 & 0.405607000 & -0.029728000 \\ \mathrm{H} & -9.946173000 & 0.166591000 & -0.049550000 \\ \mathrm{C} & -8.482185000 & 1.747196000 & 0.068237000 \\ \mathrm{H} & -9.231347000 & 2.528358000 & 0.122640000 \\ \mathrm{C} & -7.136148000 & 2.083589000 & 0.096281000 \\ \mathrm{H} & -6.813564000 & 3.115252000 & 0.172139000 \\ \mathrm{C} & -6.177153000 & 1.061208000 & 0.025291000 \\ \mathrm{C} & -4.159941000 & 0.118991000 & -0.035041000 \\ \mathrm{C} & -2.717128000 & 0.051780000 & -0.030491000 \\ \mathrm{H} & -2.239288000 & 1.016233000 & 0.048273000 \\ \mathrm{C} & -2.006249000 & -1.098451000 & -0.106831000 \\ \mathrm{H} & -2.574807000 & -2.020667000 & -0.169189000 \\ \mathrm{C} & -0.586771000 & -1.343831000 & -0.106770000 \\ \mathrm{C} & -0.043375000 & -2.658483000 & -0.109344000 \\ \mathrm{C} & -0.833850000 & -3.939999000 & -0.101373000 \\ \mathrm{H} & -0.257036000 & -4.718796000 & -0.606202000 \\ \mathrm{H} & -1.742991000 & -3.823134000 & -0.697439000 \\ \mathrm{C} & -1.209965000 & -4.426135000 & 1.310571000 \\ \mathrm{H} & -0.316828000 & -4.600655000 & 1.916434000 \\ \mathrm{H} & -1.772572000 & -5.362222000 & 1.257978000 \\ \mathrm{H} & -1.826736000 & -3.688741000 & 1.831203000 \\ \mathrm{C} & 1.347073000 & -2.543169000 & -0.085527000 \\ & 2.296450000 & -3.705259000 & -0.060000000 \\ \mathrm{H} & 1.747043000 & -4.639347000 & 0.054736000 \\ \mathrm{H} & 2.881760000 & -3.773568000 & -0.980021000 \\ & 1.643698000 & -1.146126000 & -0.089578000\end{array}$




$\begin{array}{lccc}\mathrm{C} & 2.891463000 & -0.472830000 & -0.096500000 \\ \mathrm{C} & 4.159135000 & -1.265126000 & -0.090746000 \\ \mathrm{C} & 4.726152000 & -1.700519000 & -1.292605000 \\ \mathrm{H} & 4.238452000 & -1.467273000 & -2.232473000 \\ \mathrm{C} & 5.915211000 & -2.427951000 & -1.285881000 \\ \mathrm{H} & 6.347685000 & -2.758670000 & -2.223321000 \\ \mathrm{C} & 6.545425000 & -2.729250000 & -0.078568000 \\ \mathrm{H} & 7.469087000 & -3.296634000 & -0.073788000 \\ \mathrm{C} & 5.982582000 & -2.297991000 & 1.122547000 \\ \mathrm{H} & 6.467124000 & -2.528238000 & 2.064566000 \\ \mathrm{C} & 4.795572000 & -1.567194000 & 1.117229000 \\ \mathrm{H} & 4.360931000 & -1.231988000 & 2.052264000 \\ \mathrm{C} & 2.960385000 & 0.917768000 & -0.114081000 \\ \mathrm{C} & 4.071622000 & 1.834510000 & -0.149356000 \\ \mathrm{C} & 5.536093000 & 1.520272000 & -0.159373000 \\ \mathrm{H} & 6.118279000 & 2.435121000 & -0.271836000 \\ \mathrm{H} & 5.802714000 & 0.845338000 & -0.974763000 \\ \mathrm{H} & 5.850247000 & 1.036843000 & 0.768962000 \\ \mathrm{C} & 3.532074000 & 3.116458000 & -0.175369000 \\ \mathrm{C} & 4.270540000 & 4.426922000 & -0.203990000 \\ \mathrm{H} & 3.657743000 & 5.176352000 & -0.712957000 \\ \mathrm{H} & 5.175722000 & 4.322243000 & -0.808841000 \\ \mathrm{C} & 4.654070000 & 4.949335000 & 1.192961000 \\ \mathrm{H} & 3.768045000 & 5.101941000 & 1.815271000 \\ \mathrm{H} & 5.181733000 & 5.903960000 & 1.116172000 \\ \mathrm{H} & 5.306752000 & 4.241522000 & 1.710668000 \\ \mathrm{C} & 2.117106000 & 2.983487000 & -0.150942000 \\ \mathrm{C} & 1.117446000 & 4.089818000 & -0.195449000 \\ \mathrm{H} & 0.163638000 & 3.790234000 & 0.233287000 \\ \mathrm{H} & 0.939817000 & 4.397212000 & -1.232228000 \\ \mathrm{H} & 1.490624000 & 4.962249000 & 0.342939000\end{array}$

Coordinates of the geometry of isomer ttt of $\mathbf{B T}$ optimized with the B3LYP functional

$\begin{array}{lccc}\mathrm{S} & -5.863726000 & -1.550706000 & 0.446418000 \\ \mathrm{~F} & -0.241486000 & 1.611109000 & 1.141495000 \\ \mathrm{~F} & -0.276883000 & 1.742487000 & -1.149538000 \\ \mathrm{~N} & -4.608296000 & 0.617165000 & -0.294193000 \\ \mathrm{~N} & 0.470557000 & -0.335494000 & -0.122761000 \\ \mathrm{~N} & 1.877426000 & 1.724560000 & -0.041664000 \\ \mathrm{~B} & 0.424447000 & 1.205491000 & -0.041508000 \\ \mathrm{C} & -6.815370000 & -0.121453000 & 0.118627000 \\ \mathrm{C} & -8.198076000 & 0.055899000 & 0.189039000 \\ \mathrm{H} & -8.852787000 & -0.755575000 & 0.482026000 \\ \mathrm{C} & -8.714268000 & 1.307691000 & -0.128544000 \\ \mathrm{H} & -9.784819000 & 1.469094000 & -0.081314000 \\ \mathrm{C} & -7.868045000 & 2.364184000 & -0.509206000 \\ \mathrm{H} & -8.297944000 & 3.329242000 & -0.750886000 \\ \mathrm{C} & -6.493863000 & 2.188827000 & -0.579650000 \\ \mathrm{H} & -5.835860000 & 2.998404000 & -0.872324000 \\ \mathrm{C} & -5.949469000 & 0.933451000 & -0.264115000 \\ \mathrm{C} & -4.394503000 & -0.622515000 & 0.045859000 \\ \mathrm{C} & -3.107161000 & -1.283627000 & 0.121792000 \\ \mathrm{H} & -3.127519000 & -2.323776000 & 0.414233000 \\ \mathrm{C} & -1.944891000 & -0.634638000 & -0.129610000 \\ \mathrm{H} & -2.015799000 & 0.419188000 & -0.368261000\end{array}$




\begin{tabular}{|c|c|c|}
\hline-0.610158000 & -1.177475000 & -0.110875000 \\
\hline-0.143629000 & -2.521517000 & -0.125464000 \\
\hline-0.981412000 & -3.771087000 & -0.163557000 \\
\hline-0.448433000 & -4.538252000 & -0.731253000 \\
\hline-1.899382000 & -3.580638000 & -0.725089000 \\
\hline-1.327270000 & -4.337682000 & 1.226605000 \\
\hline-0.420388000 & -4.613903000 & 1.770677000 \\
\hline-1.951540000 & -5.230480000 & 1.133609000 \\
\hline-1.866448000 & -3.609481000 & 1.838153000 \\
\hline 1.253155000 & -2.476715000 & -0.133998000 \\
\hline 2.142610000 & -3.685205000 & -0.150536000 \\
\hline 1.554415000 & -4.592475000 & -0.015506000 \\
\hline 2.888530000 & -3.651481000 & 0.644846000 \\
\hline 2.685955000 & -3.777478000 & -1.094221000 \\
\hline 1.629461000 & -1.096823000 & -0.131033000 \\
\hline 2.902256000 & -0.474316000 & -0.129376000 \\
\hline 4.135158000 & -1.318172000 & -0.165231000 \\
\hline 4.645055000 & -1.772236000 & -1.385795000 \\
\hline 4.140894000 & -1.510962000 & -2.309436000 \\
\hline 5.799697000 & -2.552365000 & -1.418451000 \\
\hline 6.188837000 & -2.895872000 & -2.370193000 \\
\hline 6.450765000 & -2.890745000 & -0.232160000 \\
\hline 7.347172000 & -3.499742000 & -0.258207000 \\
\hline 5.944018000 & -2.442483000 & 0.987555000 \\
\hline 6.444559000 & -2.701641000 & 1.913563000 \\
\hline 4.792734000 & -1.657291000 & 1.021467000 \\
\hline 4.401834000 & -1.308552000 & 1.970738000 \\
\hline 3.021608000 & 0.914405000 & -0.096757000 \\
\hline 4.167130000 & 1.786550000 & -0.118534000 \\
\hline 5.617103000 & 1.414906000 & -0.178452000 \\
\hline 6.230373000 & 2.305800000 & -0.316619000 \\
\hline 5.828837000 & 0.726267000 & -0.998108000 \\
\hline 5.945079000 & 0.924732000 & 0.741831000 \\
\hline 3.681025000 & 3.090230000 & -0.076795000 \\
\hline 4.474511000 & 4.368321000 & -0.064539000 \\
\hline 3.883995000 & 5.165622000 & -0.524705000 \\
\hline 5.360743000 & 4.252978000 & -0.695025000 \\
\hline 4.911065000 & 4.809242000 & 1.344823000 \\
\hline 4.045867000 & 4.967998000 & 1.994320000 \\
\hline 5.475848000 & 5.744448000 & 1.299045000 \\
\hline 5.545404000 & 4.052398000 & 1.813842000 \\
\hline 2.263598000 & 3.014166000 & -0.026615000 \\
\hline 1.305802000 & 4.157510000 & 0.003513000 \\
\hline 0.367647000 & 3.881783000 & 0.481410000 \\
\hline 1.079042000 & 4.490802000 & -1.015430000 \\
\hline 1.738978000 & 5.005182000 & 0.535934000 \\
\hline
\end{tabular}

Coordinates of the geometry of isomer ttc of $\mathbf{B T}$ optimized with the B3LYP functional

$\begin{array}{cccc}\mathrm{S} & -4.650354000 & 1.121186000 & -0.493673000 \\ \mathrm{~F} & -0.209604000 & 1.644887000 & 1.110773000 \\ \mathrm{~F} & -0.224448000 & 1.759312000 & -1.180344000 \\ \mathrm{~N} & -5.521615000 & -1.193752000 & 0.357320000 \\ \mathrm{~N} & 0.473465000 & -0.324673000 & -0.133188000 \\ \mathrm{~N} & 1.921855000 & 1.705547000 & -0.055156000 \\ \mathrm{~B} & 0.459213000 & 1.217110000 & -0.062866000\end{array}$




\begin{tabular}{|c|c|c|c|}
\hline & -6.372031000 & 0.925607000 & -0.244544000 \\
\hline & -7.399246000 & 1.849562000 & -0.437642000 \\
\hline & -7.192838000 & 2.853511000 & -0.787476000 \\
\hline & -8.702572000 & 1.444073000 & -0.166852000 \\
\hline & -9.517058000 & 2.144594000 & -0.309367000 \\
\hline & -8.976949000 & 0.142619000 & 0.287427000 \\
\hline & -10.001765000 & -0.145388000 & 0.490455000 \\
\hline & -7.954776000 & -0.776091000 & 0.479076000 \\
\hline & -8.157398000 & -1.781250000 & 0.829400000 \\
\hline & -6.632625000 & -0.388535000 & 0.212104000 \\
\hline & -4.420905000 & -0.568399000 & 0.038968000 \\
\hline & -3.120246000 & -1.194600000 & 0.113309000 \\
\hline & -3.161707000 & -2.224489000 & 0.440149000 \\
\hline & -1.948808000 & -0.573628000 & -0.158893000 \\
\hline & -1.979052000 & 0.475716000 & -0.429733000 \\
\hline & -0.624274000 & -1.143261000 & -0.123117000 \\
\hline & -0.187958000 & -2.496830000 & -0.122380000 \\
\hline & -1.052471000 & -3.728091000 & -0.159797000 \\
\hline & -0.537188000 & -4.505852000 & -0.729516000 \\
\hline & -1.967506000 & -3.517047000 & -0.718641000 \\
\hline & -1.408095000 & -4.288509000 & 1.230337000 \\
\hline & -0.506646000 & -4.584141000 & 1.773245000 \\
\hline & -2.051145000 & -5.167826000 & 1.137180000 \\
\hline & -1.931512000 & -3.549699000 & 1.842889000 \\
\hline & 1.209907000 & -2.482054000 & -0.119630000 \\
\hline & 2.072915000 & -3.709611000 & -0.119300000 \\
\hline & 1.462439000 & -4.604009000 & 0.001881000 \\
\hline & 2.802114000 & -3.692535000 & 0.692178000 \\
\hline & 2.634311000 & -3.812785000 & -1.051031000 \\
\hline & 1.615943000 & -1.110887000 & -0.124728000 \\
\hline & 2.901396000 & -0.515207000 & -0.115865000 \\
\hline & 4.116294000 & -1.385459000 & -0.132101000 \\
\hline & 4.630513000 & -1.858372000 & -1.343692000 \\
\hline & 4.143378000 & -1.591775000 & -2.274917000 \\
\hline & 5.767605000 & -2.664360000 & -1.357681000 \\
\hline & 6.160195000 & -3.022583000 & -2.302554000 \\
\hline & 6.396860000 & -3.009494000 & -0.161586000 \\
\hline & 7.279805000 & -3.638282000 & -0.173094000 \\
\hline & 5.885920000 & -2.542070000 & 1.049139000 \\
\hline & 6.369608000 & -2.806201000 & 1.982664000 \\
\hline & 4.752085000 & -1.731298000 & 1.064426000 \\
\hline & 4.357802000 & -1.367928000 & 2.006785000 \\
\hline & 3.049233000 & 0.871014000 & -0.093199000 \\
\hline & 4.212766000 & 1.718785000 & -0.110575000 \\
\hline & 5.655095000 & 1.316480000 & -0.153873000 \\
\hline & 6.288039000 & 2.192723000 & -0.297007000 \\
\hline & 5.859156000 & 0.614078000 & -0.963641000 \\
\hline & 5.965037000 & 0.830438000 & 0.774880000 \\
\hline & 3.753977000 & 3.032813000 & -0.083160000 \\
\hline & 4.575089000 & 4.293353000 & -0.072461000 \\
\hline & 4.005066000 & 5.101100000 & -0.540119000 \\
\hline & 5.462366000 & 4.155257000 & -0.696918000 \\
\hline & 5.012612000 & 4.731620000 & 1.337432000 \\
\hline & 4.147073000 & 4.911472000 & 1.980929000 \\
\hline & 00 & 5.654512000 & 1.29071000 \\
\hline
\end{tabular}




$\begin{array}{lllc}\mathrm{H} & 5.628142000 & 3.963901000 & 1.813780000 \\ \mathrm{C} & 2.335062000 & 2.987055000 & -0.045951000 \\ \mathrm{C} & 1.400336000 & 4.149635000 & -0.032880000 \\ \mathrm{H} & 0.458348000 & 3.900054000 & 0.452074000 \\ \mathrm{H} & 1.176604000 & 4.469668000 & -1.056697000 \\ \mathrm{H} & 1.851339000 & 4.997428000 & 0.484055000\end{array}$

Coordinates of the geometry of isomer ctt of $\mathbf{B T}$ optimized with the M06HF functional

\begin{tabular}{|c|c|c|c|}
\hline & -4.958444000 & 1.784274000 & 15000 \\
\hline & -0.279792000 & 1.553397000 & 1.118480000 \\
\hline & -0.313760000 & 1.622681000 & -1.176565000 \\
\hline & -4.925811000 & -0.797979000 & -0.060440000 \\
\hline & 0.424157000 & -0.427627000 & -0.094901000 \\
\hline & 1.820891000 & 1.641382000 & -0.068348000 \\
\hline & 0.368792000 & 1.105835000 & -0.054227000 \\
\hline & -6.485849000 & 0.959524000 & -0.043671000 \\
\hline & -7.777029000 & 1.490842000 & -0.041123000 \\
\hline & -7.939411000 & 2.558532000 & -0.030350000 \\
\hline & -8.839039000 & 0.604321000 & -0.053162000 \\
\hline & -9.849063000 & 0.987135000 & -0.051754000 \\
\hline & -8.624507000 & -0.785306000 & -0.067242000 \\
\hline & -9.475144000 & -1.451391000 & -0.076248000 \\
\hline & -7.345856000 & -1.308278000 & -0.069982000 \\
\hline & -7.165688000 & -2.373423000 & -0.081152000 \\
\hline & -6.259994000 & -0.424189000 & -0.058335000 \\
\hline & -4.148073000 & 0.225677000 & -0.048403000 \\
\hline & -2.676471000 & 0.186275000 & -0.050533000 \\
\hline & -2.165648000 & 1.135290000 & -0.028245000 \\
\hline & -2.047569000 & -0.993243000 & -0.082015000 \\
\hline & -2.672914000 & -1.877725000 & -0.096044000 \\
\hline & -0.619669000 & -1.297136000 & -0.089418000 \\
\hline & -0.125500000 & -2.611670000 & -0.079687000 \\
\hline & -0.942198000 & -3.879572000 & -0.040525000 \\
\hline & -0.372767000 & -4.678202000 & -0.513602000 \\
\hline & -1.852672000 & -3.757320000 & -0.624837000 \\
\hline & -1.299909000 & -4.280093000 & 1.405956000 \\
\hline & -0.389689000 & -4.426053000 & 1.987672000 \\
\hline & -1.877077000 & -5.204256000 & 1.413569000 \\
\hline & -1.886789000 & -3.491491000 & 1.877213000 \\
\hline & 1.274806000 & -2.534899000 & -0.057871000 \\
\hline & 2.187491000 & -3.738745000 & -0.012072000 \\
\hline & 1.654192000 & -4.568903000 & 0.447582000 \\
\hline & 3.087220000 & -3.537850000 & 0.563501000 \\
\hline & 2.483176000 & -4.030961000 & -1.020567000 \\
\hline & 1.592732000 & -1.163682000 & -0.083924000 \\
\hline & 2.866300000 & -0.518603000 & -0.105970000 \\
\hline & 4.103182000 & -1.349094000 & -0.128930000 \\
\hline & 4.518675000 & -1.943544000 & -1.317004000 \\
\hline & 3.942353000 & -1.799830000 & -2.221059000 \\
\hline & 5.677857000 & -2.711504000 & -1.330527000 \\
\hline & 6.008793000 & -3.168295000 & -2.252033000 \\
\hline & 6.407054000 & -2.894769000 & -0.158486000 \\
\hline & 7.305002000 & -3.496434000 & -0.170549000 \\
\hline & 5.982965000 & -2.304329000 & 1.028441000 \\
\hline & 6.545318000 & -2.448277000 & 1.939544000 \\
\hline
\end{tabular}




$\begin{array}{lrrc}\mathrm{C} & 4.831128000 & -1.525186000 & 1.044231000 \\ \mathrm{H} & 4.493217000 & -1.058437000 & 1.959817000 \\ \mathrm{C} & 2.971232000 & 0.842565000 & -0.109045000 \\ \mathrm{C} & 4.112592000 & 1.733398000 & -0.182245000 \\ \mathrm{C} & 5.569618000 & 1.360844000 & -0.249908000 \\ \mathrm{H} & 6.150218000 & 2.237714000 & -0.528822000 \\ \mathrm{H} & 5.735831000 & 0.568048000 & -0.977563000 \\ \mathrm{H} & 5.910844000 & 1.006988000 & 0.722814000 \\ \mathrm{C} & 3.612144000 & 3.010676000 & -0.180553000 \\ \mathrm{C} & 4.372137000 & 4.310667000 & -0.206622000 \\ \mathrm{H} & 3.720742000 & 5.097607000 & -0.583664000 \\ \mathrm{H} & 5.209007000 & 4.226427000 & -0.898152000 \\ \mathrm{C} & 4.889020000 & 4.685209000 & 1.197669000 \\ \mathrm{H} & 4.054639000 & 4.777487000 & 1.892858000 \\ \mathrm{H} & 5.426454000 & 5.631908000 & 1.162877000 \\ \mathrm{H} & 5.560854000 & 3.909673000 & 1.564934000 \\ \mathrm{C} & 2.178491000 & 2.908540000 & -0.103480000 \\ \mathrm{C} & 1.210723000 & 4.052204000 & -0.093357000 \\ \mathrm{H} & 0.223667000 & 3.723108000 & 0.214342000 \\ \mathrm{H} & 1.158206000 & 4.473625000 & -1.098799000 \\ \mathrm{H} & 1.576652000 & 4.821216000 & 0.585433000\end{array}$

Coordinates of the geometry of isomer ctc of $\mathbf{B T}$ optimized with the MO6HF functional

$\begin{array}{lccc}\text { S } & -5.162196000 & -1.315147000 & -0.161378000 \\ \mathrm{~F} & -0.302446000 & 1.499879000 & 1.163646000 \\ \mathrm{~F} & -0.401894000 & 1.570191000 & -1.130893000 \\ \mathrm{~N} & -4.774378000 & 1.232002000 & 0.088699000 \\ \mathrm{~N} & 0.438631000 & -0.451652000 & -0.074511000 \\ \mathrm{~N} & 1.758748000 & 1.666961000 & -0.076036000 \\ \mathrm{~B} & 0.328694000 & 1.078930000 & -0.029467000 \\ \mathrm{C} & -6.561886000 & -0.279943000 & -0.108600000 \\ \mathrm{C} & -7.911653000 & -0.623027000 & -0.188950000 \\ \mathrm{H} & -8.219638000 & -1.652207000 & -0.299027000 \\ \mathrm{C} & -8.841246000 & 0.400931000 & -0.126045000 \\ \mathrm{H} & -9.893888000 & 0.166099000 & -0.188019000 \\ \mathrm{C} & -8.438633000 & 1.739548000 & 0.015829000 \\ \mathrm{H} & -9.187975000 & 2.516171000 & 0.064212000 \\ \mathrm{C} & -7.100019000 & 2.074508000 & 0.094640000 \\ \mathrm{H} & -6.773551000 & 3.098532000 & 0.203189000 \\ \mathrm{C} & -6.149205000 & 1.050707000 & 0.030096000 \\ \mathrm{C} & -4.143031000 & 0.115222000 & -0.000415000 \\ \mathrm{C} & -2.677494000 & 0.044572000 & 0.027881000 \\ \mathrm{H} & -2.199492000 & 1.003023000 & 0.155518000 \\ \mathrm{C} & -2.011363000 & -1.107315000 & -0.094174000 \\ \mathrm{H} & -2.580771000 & -2.023072000 & -0.204529000 \\ \mathrm{C} & -0.571925000 & -1.358548000 & -0.085892000 \\ \mathrm{C} & -0.031292000 & -2.653979000 & -0.079379000 \\ \mathrm{C} & -0.803655000 & -3.949841000 & -0.057487000 \\ \mathrm{H} & -0.180388000 & -4.734313000 & -0.483003000 \\ \mathrm{H} & -1.684641000 & -3.874599000 & -0.694020000 \\ \mathrm{C} & -1.224489000 & -4.338309000 & 1.375026000 \\ \mathrm{H} & -0.342843000 & -4.423583000 & 2.010614000 \\ \mathrm{H} & -1.753518000 & -5.290591000 & 1.371466000 \\ \mathrm{H} & -1.877897000 & -3.572881000 & 1.794387000 \\ \mathrm{C} & 1.365374000 & -2.526317000 & -0.038649000\end{array}$




$\begin{array}{lccc}\mathrm{C} & 2.320532000 & -3.696340000 & 0.017146000 \\ \mathrm{H} & 1.818352000 & -4.539815000 & 0.487748000 \\ \mathrm{H} & 3.213870000 & -3.458371000 & 0.588565000 \\ \mathrm{H} & 2.623746000 & -3.989069000 & -0.988983000 \\ \mathrm{C} & 1.633151000 & -1.144834000 & -0.056157000 \\ \mathrm{C} & 2.882462000 & -0.453202000 & -0.085133000 \\ \mathrm{C} & 4.150026000 & -1.236357000 & -0.108265000 \\ \mathrm{C} & 4.582888000 & -1.818081000 & -1.296583000 \\ \mathrm{H} & 3.996411000 & -1.701172000 & -2.198037000 \\ \mathrm{C} & 5.771774000 & -2.538865000 & -1.313741000 \\ \mathrm{H} & 6.116025000 & -2.985259000 & -2.235560000 \\ \mathrm{C} & 6.513788000 & -2.688652000 & -0.145029000 \\ \mathrm{H} & 7.434796000 & -3.253733000 & -0.160124000 \\ \mathrm{C} & 6.072456000 & -2.111316000 & 1.042074000 \\ \mathrm{H} & 6.645221000 & -2.228377000 & 1.950569000 \\ \mathrm{C} & 4.889937000 & -1.379607000 & 1.061668000 \\ \mathrm{H} & 4.538712000 & -0.923072000 & 1.977447000 \\ \mathrm{C} & 2.937754000 & 0.910651000 & -0.106352000 \\ \mathrm{C} & 4.044895000 & 1.842661000 & -0.198017000 \\ \mathrm{C} & 5.514733000 & 1.523255000 & -0.260279000 \\ \mathrm{H} & 6.063299000 & 2.416589000 & -0.550487000 \\ \mathrm{H} & 5.710276000 & 0.727132000 & -0.976840000 \\ \mathrm{H} & 5.867355000 & 1.194814000 & 0.717297000 \\ \mathrm{C} & 3.497037000 & 3.099975000 & -0.217053000 \\ \mathrm{C} & 4.206647000 & 4.427353000 & -0.266728000 \\ \mathrm{H} & 3.526301000 & 5.180898000 & -0.661158000 \\ \mathrm{H} & 5.048787000 & 4.362446000 & -0.953902000 \\ \mathrm{C} & 4.703803000 & 4.849943000 & 1.131256000 \\ \mathrm{H} & 3.864205000 & 4.924374000 & 1.822329000 \\ \mathrm{H} & 5.204468000 & 5.815823000 & 1.077721000 \\ \mathrm{H} & 5.404095000 & 4.108579000 & 1.516158000 \\ \mathrm{C} & 2.068107000 & 2.945732000 & -0.133262000 \\ \mathrm{C} & 1.057616000 & 4.051564000 & -0.135872000 \\ & 0.085416000 & 3.689839000 & 0.182863000 \\ \mathrm{H} & 0.982880000 & 4.453879000 & -1.147737000 \\ & 1.397562000 & 4.845261000 & 0.527567000\end{array}$

Coordinates of the geometry of isomer ttt of $\mathbf{B T}$ optimized with the M06HF functional

$\begin{array}{lccc}\mathrm{S} & -5.812199000 & -1.514850000 & 0.547738000 \\ \mathrm{~F} & -0.272398000 & 1.595919000 & 1.112883000 \\ \mathrm{~F} & -0.267761000 & 1.758494000 & -1.172300000 \\ \mathrm{~N} & -4.589654000 & 0.592866000 & -0.312838000 \\ \mathrm{~N} & 0.464251000 & -0.327223000 & -0.159949000 \\ \mathrm{~N} & 1.863075000 & 1.721718000 & -0.036337000 \\ \mathrm{~B} & 0.404965000 & 1.203809000 & -0.061288000 \\ \mathrm{C} & -6.778408000 & -0.120631000 & 0.163183000 \\ \mathrm{C} & -8.161547000 & 0.048035000 & 0.250642000 \\ \mathrm{H} & -8.798636000 & -0.753239000 & 0.594754000 \\ \mathrm{C} & -8.685730000 & 1.274629000 & -0.115781000 \\ \mathrm{H} & -9.752187000 & 1.436005000 & -0.056875000 \\ \mathrm{C} & -7.852775000 & 2.315579000 & -0.563130000 \\ \mathrm{H} & -8.292524000 & 3.261892000 & -0.841807000 \\ \mathrm{C} & -6.485024000 & 2.144973000 & -0.649884000 \\ \mathrm{H} & -5.832780000 & 2.935518000 & -0.991425000 \\ \mathrm{C} & -5.938642000 & 0.909791000 & -0.281724000\end{array}$




\begin{tabular}{|c|c|c|c|}
\hline & -4.379960000 & -0.610754000 & 0.085217000 \\
\hline & -3.063658000 & -1.263362000 & 0.172985000 \\
\hline & -3.050787000 & -2.273515000 & 0.553332000 \\
\hline & -1.956106000 & -0.607650000 & -0.184140000 \\
\hline & -2.049868000 & 0.418572000 & -0.517612000 \\
\hline & -0.602489000 & -1.160971000 & -0.160280000 \\
\hline & -0.148744000 & -2.492449000 & -0.173277000 \\
\hline & -0.988685000 & -3.743720000 & -0.209938000 \\
\hline & -0.464687000 & -4.501002000 & -0.791392000 \\
\hline & -1.922291000 & -3.538334000 & -0.731448000 \\
\hline & -1.269804000 & -4.294768000 & 1.204474000 \\
\hline & -0.333273000 & -4.575874000 & 1.685416000 \\
\hline & -1.914474000 & -5.171797000 & 1.150993000 \\
\hline & -1.749547000 & -3.539480000 & 1.826957000 \\
\hline & 1.254054000 & -2.448473000 & -0.161005000 \\
\hline & 2.148266000 & -3.666498000 & -0.170326000 \\
\hline & 1.569232000 & -4.538035000 & 0.128206000 \\
\hline & 2.983969000 & -3.548676000 & 0.516549000 \\
\hline & 2.549424000 & -3.839422000 & -1.169194000 \\
\hline & 1.613161000 & -1.084303000 & -0.155473000 \\
\hline & 2.890135000 & -0.454352000 & -0.135836000 \\
\hline & 4.116068000 & -1.300533000 & -0.159873000 \\
\hline & 4.544412000 & -1.869341000 & -1.355910000 \\
\hline & 3.986553000 & -1.691935000 & -2.265674000 \\
\hline & 5.692639000 & -2.653436000 & -1.369997000 \\
\hline & 6.034034000 & -3.089909000 & -2.297664000 \\
\hline & 6.398355000 & -2.877852000 & -0.190526000 \\
\hline & 7.288390000 & -3.491250000 & -0.203058000 \\
\hline & 5.961182000 & -2.313512000 & 1.004335000 \\
\hline & 6.505795000 & -2.489752000 & 1.920587000 \\
\hline & 4.820050000 & -1.518710000 & 1.021060000 \\
\hline & 4.471998000 & -1.071340000 & 1.942583000 \\
\hline & 3.005032000 & 0.908289000 & -0.090769000 \\
\hline & 4.156004000 & 1.785425000 & -0.123345000 \\
\hline & 5.609262000 & 1.396809000 & -0.188081000 \\
\hline & 6.201885000 & 2.271140000 & -0.449464000 \\
\hline & 5.771542000 & 0.613782000 & -0.927074000 \\
\hline & 5.940226000 & 1.023831000 & 0.781148000 \\
\hline & 3.672082000 & 3.070232000 & -0.087465000 \\
\hline & 4.450181000 & 4.359753000 & -0.068754000 \\
\hline & 3.813214000 & 5.166329000 & -0.428673000 \\
\hline & 5.292050000 & 4.284035000 & -0.755288000 \\
\hline & 4.959526000 & 4.687237000 & 1.349794000 \\
\hline & 4.119840000 & 4.772720000 & 2.039504000 \\
\hline & 5.511488000 & 5.626214000 & 1.346681000 \\
\hline & 5.615922000 & 3.891442000 & 1.701176000 \\
\hline & 2.239037000 & 2.985087000 & -0.027112000 \\
\hline & 1.281750000 & 4.137335000 & 0.011396000 \\
\hline & 0.293878000 & 3.809298000 & 0.318748000 \\
\hline & 1.224926000 & 4.579228000 & -0.984989000 \\
\hline & 1.657014000 & 4.889708000 & 0.703519000 \\
\hline
\end{tabular}

Coordinates of the geometry of isomer ttc of $\mathbf{B T}$ optimized with the M06HF functional

$\mathrm{S} \quad-4.652151000 \quad 0.991425000 \quad-0.694836000$

F $\quad-0.284499000 \quad 1.616741000 \quad 1.034158000$ 


\begin{tabular}{|c|c|c|c|}
\hline 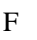 & -0.212875000 & 1.751679000 & -1.250109000 \\
\hline $\mathrm{N}$ & -5.447673000 & -1.137151000 & 0.544898000 \\
\hline & 0.469098000 & -0.328391000 & -0.193204000 \\
\hline & 1.886459000 & 1.704904000 & -0.053976000 \\
\hline & 0.424486000 & 1.203653000 & -0.114041000 \\
\hline & -6.343881000 & 0.859579000 & -0.310009000 \\
\hline & -7.378211000 & 1.756232000 & -0.579902000 \\
\hline & -7.188497000 & 2.687287000 & -1.093121000 \\
\hline $\mathrm{C}$ & -8.653342000 & 1.412594000 & -0.164534000 \\
\hline $\mathrm{H}$ & -9.473620000 & 2.087990000 & -0.359164000 \\
\hline $\mathrm{C}$ & -8.897640000 & 0.201326000 & 0.504782000 \\
\hline $\mathrm{H}$ & -9.903705000 & -0.037947000 & 0.816948000 \\
\hline $\mathrm{C}$ & -7.870417000 & -0.685082000 & 0.768241000 \\
\hline $\mathrm{H}$ & -8.042321000 & -1.620949000 & 1.279773000 \\
\hline $\mathrm{C}$ & -6.576525000 & -0.351187000 & 0.354123000 \\
\hline $\mathrm{C}$ & -4.395183000 & -0.576469000 & 0.064971000 \\
\hline$C$ & -3.066788000 & -1.194895000 & 0.151071000 \\
\hline $\mathrm{H}$ & -3.069106000 & -2.178195000 & 0.597341000 \\
\hline C & -1.953164000 & -0.578120000 & -0.251495000 \\
\hline $\mathrm{H}$ & -2.006614000 & 0.430947000 & -0.645050000 \\
\hline $\mathrm{C}$ & -0.607067000 & -1.149197000 & -0.196496000 \\
\hline C & -0.171781000 & -2.485582000 & -0.181507000 \\
\hline C & -1.029979000 & -3.724556000 & -0.208959000 \\
\hline $\mathrm{H}$ & -0.522530000 & -4.490369000 & -0.79 \\
\hline $\mathrm{H}$ & -1.965935000 & -3.504580000 & -0.720329000 \\
\hline $\mathrm{C}$ & -1.307374000 & -4.269257000 & 1.208538000 \\
\hline $\mathrm{H}$ & -0.371140000 & -4.557238000 & 1.686015000 \\
\hline $\mathrm{H}$ & -1.959986000 & -5.140631000 & 1.160486000 \\
\hline $\mathrm{H}$ & -1.778072000 & -3.508133000 & 1.830702000 \\
\hline $\mathrm{C}$ & 1.231968000 & -2.458637000 & -0.152491000 \\
\hline $\mathrm{C}$ & 2.110448000 & -3.687907000 & 324000 \\
\hline $\mathrm{H}$ & 1.514348000 & -4.550621000 & 0.154099000 \\
\hline $\mathrm{H}$ & 2.933252000 & -3.576608000 & 0.567121000 \\
\hline $\mathrm{H}$ & 2.529915000 & -3.870893000 & -1.125799000 \\
\hline r & 1.608319000 & -1.099397000 & -0.161192000 \\
\hline $\mathrm{C}$ & 2.892010000 & -0.483070000 & -0.123202000 \\
\hline $\mathrm{C}$ & 4.107082000 & -1.345000000 & -0.119904000 \\
\hline $\mathrm{C}$ & 4.545994000 & -1.928911000 & -1.304888000 \\
\hline $\mathrm{H}$ & 4.005593000 & -1.749565000 & -2.224708000 \\
\hline $\mathrm{C}$ & 5.680606000 & -2.732425000 & -1.294564000 \\
\hline $\mathrm{H}$ & 6.029589000 & -3.181092000 & -2.213474000 \\
\hline $\mathrm{C}$ & 6.363121000 & -2.960181000 & -0.102136000 \\
\hline $\mathrm{H}$ & 7.242699000 & -3.588570000 & -0.096179000 \\
\hline $\mathrm{C}$ & 5.916078000 & -2.379687000 & 1.081333000 \\
\hline $\mathrm{H}$ & 6.443194000 & -2.557760000 & 2.007398000 \\
\hline$r$ & 4.788506000 & -1.565582000 & 1.073751000 \\
\hline $\mathrm{H}$ & 4.432763000 & -1.106626000 & 1.986496000 \\
\hline $\mathrm{C}$ & 3.020453000 & 0.878512000 & -0.083110000 \\
\hline $\mathrm{C}$ & 4.181252000 & 1.743271000 & -0.093616000 \\
\hline C & 5.631051000 & 1.338400000 & -0.126842000 \\
\hline$\Pi$ & 6.238273000 & 2.204609000 & -0.381747000 \\
\hline $\mathrm{H}$ & 5.799372000 & 0.549157000 & -0.857737000 \\
\hline $\mathrm{H}$ & 5.938579000 & 0.968159000 & 0.851033000 \\
\hline 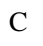 & 3.710936000 & 3.033384000 & -0.069449000 \\
\hline & 4.501679000 & 4.314773000 & -0.032865000 \\
\hline
\end{tabular}




$\begin{array}{lccc}\mathrm{H} & 3.882030000 & 5.127600000 & -0.408640000 \\ \mathrm{H} & 5.359566000 & 4.230141000 & -0.698284000 \\ \mathrm{C} & 4.979222000 & 4.637848000 & 1.397821000 \\ \mathrm{H} & 4.123866000 & 4.727538000 & 2.067593000 \\ \mathrm{H} & 5.536692000 & 5.573517000 & 1.408781000 \\ \mathrm{H} & 5.622358000 & 3.837769000 & 1.763606000 \\ \mathrm{C} & 2.276354000 & 2.964084000 & -0.039526000 \\ \mathrm{C} & 1.331061000 & 4.126697000 & -0.025266000 \\ \mathrm{H} & 0.333446000 & 3.810438000 & 0.262385000 \\ \mathrm{H} & 1.300649000 & 4.565283000 & -1.024230000 \\ \mathrm{H} & 1.699775000 & 4.877646000 & 0.671898000\end{array}$

Coordinates of the geometry of isomer ctt of NBT optimized with the B3LYP functional

\begin{tabular}{|c|c|c|c|}
\hline & -4.255738000 & 281000 & 9000 \\
\hline$F$ & 0.456274000 & 1.512764000 & 1.087770000 \\
\hline 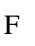 & 0.403138000 & 1.532337000 & -1.211314000 \\
\hline v & -4.140079000 & -1.096916000 & -0.090653000 \\
\hline $\mathrm{N}$ & 1.232719000 & -0.463920000 & 699000 \\
\hline & 2.548985000 & 1.669037000 & -0.112282000 \\
\hline B & 1.124053000 & 1.075700000 & -0.083618000 \\
\hline $\mathrm{C}$ & -5.755656000 & 0.621120000 & 0.000658000 \\
\hline C & -7.057464000 & 1.098706000 & 0.023511000 \\
\hline $\mathrm{H}$ & -7.288941000 & 2.152539000 & 0.080367000 \\
\hline $\mathrm{C}$ & -8.077694000 & 0.151618000 & -0.031084000 \\
\hline $\mathrm{C}$ & -7.831162000 & -1.230052000 & -0.105498000 \\
\hline $\mathrm{H}$ & -8.664905000 & -1.916046000 & 819000 \\
\hline $\mathrm{C}$ & -6.530013000 & -1.691683000 & -0.127332000 \\
\hline $\mathrm{H}$ & -6.316995000 & -2.751551000 & -0.184194000 \\
\hline $\mathrm{C}$ & -5.467969000 & -0.770080000 & -0.074877000 \\
\hline C & -3.376747000 & -0.033319000 & -0.030987000 \\
\hline $\mathrm{C}$ & -1.934594000 & -0.009566000 & -0.026966000 \\
\hline $\mathrm{H}$ & -1.469065000 & 0.961519000 & 0.031410000 \\
\hline $\mathrm{C}$ & -1.206665000 & -1.154176000 & -0.088063000 \\
\hline $\mathrm{H}$ & -1.782718000 & -2.071337000 & -0.132226000 \\
\hline $\mathrm{C}$ & 0.210959000 & -1.382850000 & 058000 \\
\hline $\mathrm{C}$ & 0.770507000 & -2.690547000 & -0.099413000 \\
\hline $\mathrm{C}$ & -0.001955000 & -3.982429000 & -0.082928000 \\
\hline $\mathrm{H}$ & 0.570030000 & -4.747390000 & -0.614013000 \\
\hline $\mathrm{H}$ & -0.930926000 & -3.871095000 & -0.647965000 \\
\hline $\mathrm{C}$ & -0.326670000 & -4.488208000 & 1.334841000 \\
\hline $\mathrm{H}$ & 0.586820000 & -4.656082000 & 1.911509000 \\
\hline $\mathrm{H}$ & -0.877259000 & -5.431705000 & 1.289215000 \\
\hline $\mathrm{H}$ & -0.937799000 & -3.764873000 & 1.881065000 \\
\hline $\mathrm{C}$ & 2.160155000 & -2.557798000 & -0.085872000 \\
\hline $\mathrm{C}$ & 3.124257000 & -3.707915000 & -0.067291000 \\
\hline $\mathrm{H}$ & 2.585380000 & -4.650483000 & 0.025669000 \\
\hline $\mathrm{H}$ & 3.822786000 & -3.641258000 & 0.768150000 \\
\hline $\mathrm{H}$ & 3.721979000 & -3.754016000 & -0.980378000 \\
\hline $\mathrm{C}$ & 2.437629000 & -1.157451000 & -0.091778000 \\
\hline $\mathrm{C}$ & 3.681537000 & -0.468297000 & -0.103013000 \\
\hline $\mathrm{C}$ & 4.957220000 & -1.247324000 & -0.105699000 \\
\hline $\mathrm{C}$ & 5.526126000 & -1.666468000 & -1.312447000 \\
\hline $\mathrm{H}$ & 5.034363000 & -1.430298000 & -2.249454000 \\
\hline $\mathrm{C}$ & 6.722364000 & -2.381984000 & -1.313766000 \\
\hline & 7.156535000 & -2.700260000 & -2.254686000 \\
\hline
\end{tabular}




$\begin{array}{lccc}\mathrm{C} & 7.357636000 & -2.687096000 & -0.110075000 \\ \mathrm{H} & 8.287018000 & -3.245042000 & -0.111740000 \\ \mathrm{C} & 6.792652000 & -2.272017000 & 1.095669000 \\ \mathrm{H} & 7.281232000 & -2.505275000 & 2.034823000 \\ \mathrm{C} & 5.598237000 & -1.553363000 & 1.098764000 \\ \mathrm{H} & 5.161886000 & -1.230723000 & 2.037398000 \\ \mathrm{C} & 3.733134000 & 0.919779000 & -0.116351000 \\ \mathrm{C} & 4.835576000 & 1.851538000 & -0.151046000 \\ \mathrm{C} & 6.302613000 & 1.552548000 & -0.164774000 \\ \mathrm{H} & 6.875402000 & 2.474167000 & -0.268837000 \\ \mathrm{H} & 6.574769000 & 0.887593000 & -0.986487000 \\ \mathrm{H} & 6.621219000 & 1.063234000 & 0.758877000 \\ \mathrm{C} & 4.281905000 & 3.124901000 & -0.171274000 \\ \mathrm{C} & 5.002442000 & 4.445066000 & -0.195923000 \\ \mathrm{H} & 4.379276000 & 5.187516000 & -0.702412000 \\ \mathrm{H} & 5.908840000 & 4.354238000 & -0.800980000 \\ \mathrm{C} & 5.378679000 & 4.967390000 & 1.203105000 \\ \mathrm{H} & 4.491160000 & 5.104499000 & 1.826810000 \\ \mathrm{H} & 5.891876000 & 5.929985000 & 1.129239000 \\ \mathrm{H} & 6.042340000 & 4.267424000 & 1.717381000 \\ \mathrm{C} & 2.866148000 & 2.974844000 & -0.144100000 \\ \mathrm{C} & 1.856695000 & 4.071349000 & -0.184186000 \\ \mathrm{H} & 0.895949000 & 3.752796000 & 0.213424000 \\ \mathrm{H} & 1.703429000 & 4.402264000 & -1.217653000 \\ \mathrm{H} & 2.209228000 & 4.934176000 & 0.383155000 \\ \mathrm{~N} & -9.460728000 & 0.620132000 & -0.009552000 \\ \mathrm{O} & -10.362147000 & -0.216607000 & -0.061171000 \\ \mathrm{O} & -9.668060000 & 1.831964000 & 0.059135000\end{array}$

Coordinates of the geometry of isomer ctc of NBT optimized with the B3LYP functional

$\begin{array}{cccc}\text { S } & -4.403248000 & -1.332816000 & -0.134677000 \\ \mathrm{~F} & 0.479728000 & 1.553257000 & 1.083291000 \\ \mathrm{~F} & 0.425365000 & 1.570238000 & -1.216321000 \\ \mathrm{~N} & -4.022793000 & 1.253064000 & 0.049463000 \\ \mathrm{~N} & 1.230254000 & -0.434486000 & -0.096844000 \\ \mathrm{~N} & 2.572847000 & 1.682237000 & -0.117943000 \\ \mathrm{~B} & 1.140523000 & 1.106916000 & -0.088340000 \\ \mathrm{C} & -5.803289000 & -0.289244000 & -0.060818000 \\ \mathrm{C} & -7.146038000 & -0.634321000 & -0.083634000 \\ \mathrm{H} & -7.483156000 & -1.658194000 & -0.155395000 \\ \mathrm{C} & -8.065578000 & 0.410044000 & -0.008527000 \\ \mathrm{C} & -7.682716000 & 1.758006000 & 0.085494000 \\ \mathrm{H} & -8.443054000 & 2.523534000 & 0.140146000 \\ \mathrm{C} & -6.340725000 & 2.085941000 & 0.106868000 \\ \mathrm{H} & -6.021333000 & 3.117781000 & 0.179152000 \\ \mathrm{C} & -5.379239000 & 1.062763000 & 0.034621000 \\ \mathrm{C} & -3.367716000 & 0.119535000 & -0.029401000 \\ \mathrm{C} & -1.929104000 & 0.058721000 & -0.027871000 \\ \mathrm{H} & -1.453109000 & 1.024248000 & 0.045572000 \\ \mathrm{C} & -1.217575000 & -1.093847000 & -0.100768000 \\ \mathrm{H} & -1.786872000 & -2.016075000 & -0.157881000 \\ \mathrm{C} & 0.198171000 & -1.340983000 & -0.103291000 \\ \mathrm{C} & 0.741088000 & -2.655268000 & -0.105229000 \\ \mathrm{C} & -0.047248000 & -3.937748000 & -0.093672000 \\ \mathrm{H} & 0.524584000 & -4.712622000 & -0.610065000\end{array}$




\begin{tabular}{|c|c|c|}
\hline-0.964362000 & -3.820038000 & -0.676949000 \\
\hline-0.403161000 & -4.431198000 & 1.321024000 \\
\hline 0.498082000 & -4.606348000 & 1.914470000 \\
\hline-0.963931000 & -5.368415000 & 1.270919000 \\
\hline-1.015031000 & -3.697779000 & 1.852860000 \\
\hline 2.132297000 & -2.539397000 & -0.083426000 \\
\hline 3.081707000 & -3.701523000 & -0.058367000 \\
\hline 2.531580000 & -4.635762000 & 0.051096000 \\
\hline 3.788383000 & -3.633528000 & 0.769826000 \\
\hline 3.670378000 & -3.766918000 & -0.976313000 \\
\hline 2.426829000 & -1.142784000 & -0.088999000 \\
\hline 3.679120000 & -0.468623000 & -0.098069000 \\
\hline 4.945184000 & -1.263206000 & -0.093187000 \\
\hline 5.511270000 & -1.697616000 & -1.295822000 \\
\hline 5.024475000 & -1.461661000 & -2.235463000 \\
\hline 6.698682000 & -2.427660000 & -1.289751000 \\
\hline 7.130832000 & -2.757610000 & -2.227576000 \\
\hline 7.327721000 & -2.732327000 & -0.082691000 \\
\hline 8.250161000 & -3.301664000 & -0.078578000 \\
\hline 6.765498000 & -2.301991000 & 1.119011000 \\
\hline 7.249279000 & -2.534781000 & 2.060761000 \\
\hline 5.580038000 & -1.568728000 & 1.114711000 \\
\hline 5.145779000 & -1.234280000 & 2.050174000 \\
\hline 3.747731000 & 0.918470000 & -0.116579000 \\
\hline 4.861640000 & 1.836648000 & -0.152967000 \\
\hline 6.324881000 & 1.519659000 & -0.164173000 \\
\hline 6.909035000 & 2.433878000 & -0.270347000 \\
\hline 6.589572000 & 0.849165000 & -0.983879000 \\
\hline 6.636575000 & 1.029000000 & 0.761088000 \\
\hline 4.323620000 & 3.116466000 & -0.178976000 \\
\hline 5.060458000 & 4.427514000 & -0.207762000 \\
\hline 4.446708000 & 5.175945000 & -0.716945000 \\
\hline 5.965851000 & 4.323294000 & -0.812159000 \\
\hline 5.442573000 & 4.949794000 & 1.189715000 \\
\hline 4.556502000 & 5.099954000 & 1.812462000 \\
\hline 5.967674000 & 5.905705000 & 1.112859000 \\
\hline 6.097291000 & 4.243379000 & 1.706636000 \\
\hline 2.905942000 & 2.983827000 & -0.153629000 \\
\hline 1.910298000 & 4.092671000 & -0.198069000 \\
\hline 0.943956000 & 3.786344000 & 0.195555000 \\
\hline 1.765565000 & 4.425394000 & -1.232180000 \\
\hline 2.271322000 & 4.951097000 & 0.370681000 \\
\hline-9.490001000 & 0.082643000 & -0.028633000 \\
\hline-10.302014000 & 1.004561000 & 0.042413000 \\
\hline-9.817428000 & -1.100784000 & -0.115366000 \\
\hline
\end{tabular}

Coordinates of the geometry of isomer ttt of NBT optimized with the B3LYP functional

$\begin{array}{cccc}\mathrm{S} & -4.983254000 & -1.989352000 & 0.368851000 \\ \mathrm{~F} & 0.437868000 & 1.510515000 & 1.125974000 \\ \mathrm{~F} & 0.391120000 & 1.632121000 & -1.164945000 \\ \mathrm{~N} & -3.834946000 & 0.277124000 & -0.257376000 \\ \mathrm{~N} & 1.267276000 & -0.391691000 & -0.133410000 \\ \mathrm{~N} & 2.543644000 & 1.752039000 & -0.060052000 \\ \mathrm{~B} & 1.124700000 & 1.144310000 & -0.056760000 \\ \mathrm{C} & -5.998153000 & -0.593154000 & 0.105450000\end{array}$




\begin{tabular}{|c|c|c|c|}
\hline & -7.379321000 & -0.491777000 & 1000 \\
\hline & -8.011753000 & -1.331894000 & 0.426015000 \\
\hline & -7.934068000 & 0.758693000 & -0.084399000 \\
\hline & -7.157366000 & 1.883671000 & -0.409933000 \\
\hline & -7.646094000 & 2.827614000 & -0.603101000 \\
\hline & -5.783019000 & 1.768360000 & -0.478306000 \\
\hline & -5.164815000 & 2.621587000 & -0.726710000 \\
\hline & -5.180165000 & 0.523582000 & -0.220905000 \\
\hline & -3.562780000 & -0.972103000 & 0.024763000 \\
\hline & -2.248662000 & -1.567281000 & 0.079507000 \\
\hline & -2.213793000 & -2.618581000 & 0.324798000 \\
\hline & -1.122562000 & -0.840670000 & -0.135832000 \\
\hline & -1.256789000 & 0.215968000 & -0.330525000 \\
\hline & 0.239215000 & -1.300838000 & -0.127764000 \\
\hline & 0.789313000 & -2.612610000 & -0.149736000 \\
\hline & 0.032796000 & -3.912509000 & -0.194244000 \\
\hline & 0.620151000 & -4.645309000 & -0.753183000 \\
\hline & -0.888227000 & -3.782858000 & -0.767819000 \\
\hline & -0.291550000 & -4.495852000 & 1.194198000 \\
\hline & 0.625364000 & -4.709352000 & 1.749481000 \\
\hline & -0.853926000 & -5.428373000 & 1.097255000 \\
\hline & -0.885095000 & -3.803159000 & 1.796516000 \\
\hline & 2.180864000 & -2.479549000 & -0.153215000 \\
\hline & 3.146411000 & -3.628065000 & -0.176187000 \\
\hline & 2.616714000 & -4.573733000 & -0.064316000 \\
\hline & 3.878071000 & -3.560226000 & 0.630206000 \\
\hline & 3.706967000 & -3.668423000 & -1.113261000 \\
\hline & 2.467629000 & -1.078985000 & -0.140379000 \\
\hline & 3.703561000 & -0.377806000 & -0.127382000 \\
\hline & 4.985622000 & -1.145394000 & -0.147095000 \\
\hline & 5.545207000 & -1.556672000 & -1.360931000 \\
\hline & 5.043859000 & -1.318684000 & -2.292365000 \\
\hline & 6.745147000 & -2.265790000 & -1.376313000 \\
\hline & 7.172689000 & -2.577020000 & -2.322610000 \\
\hline & 7.392174000 & -2.574534000 & -0.179829000 \\
\hline & 8.323949000 & -3.128329000 & -0.192616000 \\
\hline & 6.835716000 & -2.168768000 & 1.033025000 \\
\hline & 7.333079000 & -2.405417000 & 1.966705000 \\
\hline & 5.638650000 & -1.454767000 & 1.050201000 \\
\hline & 5.209044000 & -1.138885000 & 1.994199000 \\
\hline & 3.736415000 & 1.012301000 & -0.095969000 \\
\hline & 4.828535000 & 1.954939000 & -0.107554000 \\
\hline & 6.298387000 & 1.670758000 & -0.141848000 \\
\hline & 6.859921000 & 2.597814000 & -0.258803000 \\
\hline & 6.565866000 & 1.004049000 & -0.963333000 \\
\hline & 6.635656000 & 1.190723000 & 0.780303000 \\
\hline & 4.263498000 & 3.224197000 & -0.082378000 \\
\hline & 4.973104000 & 4.550457000 & -0.067845000 \\
\hline & 4.340943000 & 5.303953000 & -0.545995000 \\
\hline & 5.876396000 & 4.487435000 & -0.681110000 \\
\hline & 5.354162000 & 5.029733000 & 1.345083000 \\
\hline & 4.469234000 & 5.140136000 & 1.977741000 \\
\hline & 5.860111000 & 5.997948000 & 1.299712000 \\
\hline & 6.025816000 & 4.318332000 & 1.832660000 \\
\hline & 2.850376000 & 0 & -0.050260000 \\
\hline
\end{tabular}




$\begin{array}{lccc}\mathrm{C} & 1.827425000 & 4.145775000 & -0.043887000 \\ \mathrm{H} & 0.887708000 & 3.811530000 & 0.390848000 \\ \mathrm{H} & 1.625636000 & 4.479541000 & -1.068017000 \\ \mathrm{H} & 2.191023000 & 5.009644000 & 0.514226000 \\ \mathrm{~N} & -9.386365000 & 0.901332000 & -0.016946000 \\ \mathrm{O} & -9.876416000 & 2.005526000 & -0.252406000 \\ \mathrm{O} & -10.059416000 & -0.088120000 & 0.272429000\end{array}$

Coordinates of the geometry of isomer ttc of NBT optimized with the B3LYP functional

\begin{tabular}{|c|c|c|c|}
\hline & -3.957038000 & 0.593040000 & -0.341890000 \\
\hline & 0.411522000 & 1.478413000 & 1.139997000 \\
\hline & 0.356984000 & 1.600790000 & -1.149720000 \\
\hline & -4.635773000 & -1.846344000 & 0.331336000 \\
\hline $\mathrm{N}$ & 1.256720000 & -0.413994000 & -0.122834000 \\
\hline $\mathrm{N}$ & 2.512723000 & 1.741290000 & -0.051570000 \\
\hline & 1.100519000 & 1.120277000 & -0.044064000 \\
\hline C & -5.653198000 & 0.231860000 & -0.122349000 \\
\hline C & -6.745498000 & 1.075259000 & -0.258749000 \\
\hline $\mathrm{H}$ & -6.646860000 & 2.115927000 & -0.531370000 \\
\hline C & -8.001902000 & 0.519549000 & -0.027285000 \\
\hline $\mathrm{C}$ & -8.187179000 & -0.826230000 & 0.328985000 \\
\hline $\mathrm{H}$ & -9.187533000 & -1.198461000 & 0.495779000 \\
\hline $\mathrm{C}$ & -7.088945000 & -1.654031000 & 0.461174000 \\
\hline $\mathrm{H}$ & -7.206451000 & -2.694669000 & 0.735257000 \\
\hline $\mathrm{C}$ & -5.802568000 & -1.134056000 & 0.235700000 \\
\hline C & -3.588095000 & -1.104079000 & 0.067447000 \\
\hline $\mathrm{C}$ & -2.244556000 & -1.623349000 & 0.107515000 \\
\hline $\mathrm{H}$ & -2.200042000 & -2.672365000 & 0.363634000 \\
\hline C & -1.129756000 & -0.885749000 & -0.119521000 \\
\hline $\mathrm{H}$ & -1.255081000 & 0.17 & 000 \\
\hline $\mathrm{C}$ & 0.237681000 & -1.332539000 & -0.114532000 \\
\hline $\mathrm{C}$ & 0.798655000 & -2.639180000 & -0.138939000 \\
\hline C & 0.054452000 & -3.946206000 & -0.183021000 \\
\hline $\mathrm{H}$ & 0.645140000 & -4.670871000 & 0000 \\
\hline $\mathrm{H}$ & -0.872064000 & -3.823501000 & -0.749138000 \\
\hline $\mathrm{C}$ & -0.254392000 & -4.538102000 & 1.205267000 \\
\hline $\mathrm{H}$ & 0.668257000 & -4.742724000 & 1.754391000 \\
\hline$H$ & -0.806400000 & -5.476755000 & 1.108194000 \\
\hline $\mathrm{H}$ & -0.852780000 & -3.854723000 & 1.813405000 \\
\hline $\mathrm{C}$ & 2.189269000 & -2.493199000 & -0.147001000 \\
\hline $\mathrm{C}$ & 3.165137000 & -3.632822000 & -0.174542000 \\
\hline $\mathrm{H}$ & 2.644337000 & -4.583742000 & -0.065558000 \\
\hline $\mathrm{H}$ & 3.896700000 & -3.560854000 & 0.631638000 \\
\hline $\mathrm{H}$ & 3.725397000 & -3.664714000 & -1.112067000 \\
\hline C & 2.463429000 & -1.090435000 & -0.134290000 \\
\hline $\mathrm{C}$ & 3.692616000 & -0.377603000 & -0.125497000 \\
\hline $\mathrm{C}$ & 4.981599000 & -1.133287000 & -0.151047000 \\
\hline $\mathrm{C}$ & 5.539732000 & -1.538608000 & -1.367568000 \\
\hline 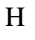 & 5.032376000 & -1.304430000 & -2.296713000 \\
\hline $\mathrm{C}$ & 6.745755000 & -2.237161000 & -1.388454000 \\
\hline $\mathrm{H}$ & 7.172110000 & -2.543892000 & -2.336750000 \\
\hline$r$ & 7.400333000 & -2.541207000 & -0.194875000 \\
\hline $\mathrm{H}$ & 8.336863000 & -3.086796000 & -0.211974000 \\
\hline $\mathrm{C}$ & 6.845364000 & -2.141334000 & 1.020602000 \\
\hline & 7.348625000 & -2.374390000 & 1.952017000 \\
\hline
\end{tabular}




$\begin{array}{cccc}\mathrm{C} & 5.642194000 & -1.437805000 & 1.043317000 \\ \mathrm{H} & 5.213728000 & -1.126490000 & 1.989342000 \\ \mathrm{C} & 3.712338000 & 1.012758000 & -0.092902000 \\ \mathrm{C} & 4.795273000 & 1.965715000 & -0.108279000 \\ \mathrm{C} & 6.267589000 & 1.695428000 & -0.148364000 \\ \mathrm{H} & 6.819773000 & 2.627308000 & -0.271238000 \\ \mathrm{H} & 6.537697000 & 1.028114000 & -0.968426000 \\ \mathrm{H} & 6.613498000 & 1.222218000 & 0.774152000 \\ \mathrm{C} & 4.218420000 & 3.229691000 & -0.079956000 \\ \mathrm{C} & 4.915690000 & 4.562506000 & -0.067465000 \\ \mathrm{H} & 4.274499000 & 5.310442000 & -0.542263000 \\ \mathrm{H} & 5.816764000 & 4.508417000 & -0.684818000 \\ \mathrm{C} & 5.298586000 & 5.044229000 & 1.344110000 \\ \mathrm{H} & 4.415496000 & 5.146185000 & 1.980739000 \\ \mathrm{H} & 5.795529000 & 6.017017000 & 1.297152000 \\ \mathrm{H} & 5.978823000 & 4.338653000 & 1.828224000 \\ \mathrm{C} & 2.807192000 & 3.053133000 & -0.042129000 \\ \mathrm{C} & 1.773562000 & 4.127781000 & -0.031137000 \\ \mathrm{H} & 0.842271000 & 3.786689000 & 0.416434000 \\ \mathrm{H} & 1.556550000 & 4.452611000 & -1.055013000 \\ \mathrm{H} & 2.134445000 & 4.998811000 & 0.517347000 \\ \mathrm{~N} & -9.176697000 & 1.379456000 & -0.164366000 \\ \mathrm{O} & -10.286262000 & 0.887849000 & 0.038242000 \\ \mathrm{O} & -9.006195000 & 2.557666000 & -0.476565000\end{array}$

Coordinates of the geometry of isomer ctt of NBT optimized with the MO6HF functional

$\begin{array}{cccc}\text { S } & -4.235882000 & 1.506658000 & -0.007981000 \\ \mathrm{~F} & 0.440238000 & 1.474693000 & 1.129737000 \\ \mathrm{~F} & 0.386127000 & 1.544417000 & -1.164900000 \\ \mathrm{~N} & -4.103431000 & -1.074944000 & -0.049430000 \\ \mathrm{~N} & 1.223144000 & -0.469925000 & -0.090629000 \\ \mathrm{~N} & 2.526085000 & 1.658521000 & -0.071406000 \\ \mathrm{~B} & 1.099907000 & 1.058655000 & -0.048521000 \\ \mathrm{C} & -5.724529000 & 0.620441000 & -0.025488000 \\ \mathrm{C} & -7.028852000 & 1.101627000 & -0.021721000 \\ \mathrm{H} & -7.263215000 & 2.155441000 & -0.004965000 \\ \mathrm{C} & -8.022512000 & 0.146002000 & -0.040462000 \\ \mathrm{C} & -7.789452000 & -1.233599000 & -0.062750000 \\ \mathrm{H} & -8.625119000 & -1.915490000 & -0.076568000 \\ \mathrm{C} & -6.489905000 & -1.689765000 & -0.065788000 \\ \mathrm{H} & -6.266565000 & -2.745931000 & -0.082222000 \\ \mathrm{C} & -5.444139000 & -0.757250000 & -0.047792000 \\ \mathrm{C} & -3.367512000 & -0.016205000 & -0.030227000 \\ \mathrm{C} & -1.899206000 & 0.009365000 & -0.029462000 \\ \mathrm{H} & -1.431519000 & 0.979915000 & 0.010599000 \\ \mathrm{C} & -1.220838000 & -1.142953000 & -0.078935000 \\ \mathrm{H} & -1.807656000 & -2.053050000 & -0.108246000 \\ \mathrm{C} & 0.216891000 & -1.384775000 & -0.087461000 \\ \mathrm{C} & 0.767561000 & -2.675343000 & -0.079968000 \\ \mathrm{C} & 0.008492000 & -3.978423000 & -0.042075000 \\ \mathrm{H} & 0.616170000 & -4.751025000 & -0.510372000 \\ \mathrm{H} & -0.904021000 & -3.899652000 & -0.630997000 \\ \mathrm{C} & -0.336753000 & -4.391733000 & 1.403870000 \\ \mathrm{H} & 0.576075000 & -4.487054000 & 1.992033000 \\ \mathrm{H} & -0.864971000 & -5.344422000 & 1.411413000\end{array}$




\begin{tabular}{|c|c|c|c|}
\hline & -0.967673000 & -3.633493000 & 1.867907000 \\
\hline & 2.164518000 & -2.536886000 & -0.056719000 \\
\hline & 3.128990000 & -3.699868000 & -0.013231000 \\
\hline & 2.632636000 & -4.553330000 & 0.444931000 \\
\hline & 4.019339000 & -3.462219000 & 0.563278000 \\
\hline & 3.437005000 & -3.977466000 & -1.022176000 \\
\hline & 2.420258000 & -1.154135000 & -0.079748000 \\
\hline & 3.667511000 & -0.451801000 & -0.101928000 \\
\hline & 4.939665000 & -1.227131000 & -0.128229000 \\
\hline & 5.377152000 & -1.800306000 & -1.318992000 \\
\hline & 4.791651000 & -1.681404000 & -2.220794000 \\
\hline & 6.569390000 & -2.515475000 & -1.337907000 \\
\hline & 6.917406000 & -2.955200000 & -2.261561000 \\
\hline & 7.310048000 & -2.668205000 & -0.168704000 \\
\hline & 8.233544000 & -3.229097000 & -0.185251000 \\
\hline & 6.864086000 & -2.099391000 & 1.020791000 \\
\hline & 7.435747000 & -2.218889000 & 1.929651000 \\
\hline & 5.678094000 & -1.373452000 & 1.042320000 \\
\hline & 5.322840000 & -0.923985000 & 1.960098000 \\
\hline & 3.711686000 & 0.910581000 & -0.106946000 \\
\hline & 4.813113000 & 1.853779000 & -0.183682000 \\
\hline & 6.285044000 & 1.544706000 & -0.243138000 \\
\hline & 6.828735000 & 2.442800000 & -0.527405000 \\
\hline & 6.487611000 & 0.752797000 & -0.962383000 \\
\hline & 6.636269000 & 1.214086000 & 0.734233000 \\
\hline & 4.255956000 & 3.105625000 & -0.190120000 \\
\hline & 4.953357000 & 4.439834000 & -0.220220000 \\
\hline & 4.271286000 & 5.190204000 & -0.617760000 \\
\hline & 5.805369000 & 4.388198000 & -0.896182000 \\
\hline & 5.427885000 & 4.855112000 & 1.187902000 \\
\hline & 4.578793000 & 4.915748000 & 1.868714000 \\
\hline & 5.919940000 & 5.826005000 & 1.148836000 \\
\hline & 6.130217000 & 4.116991000 & 1.575323000 \\
\hline & 2.826212000 & 2.938964000 & -0.113943000 \\
\hline & 1.808840000 & 4.037809000 & -0.113327000 \\
\hline & 0.837201000 & 3.667941000 & 0.197281000 \\
\hline & 1.738204000 & 4.446448000 & -1.123041000 \\
\hline & 2.140669000 & 4.828905000 & 0.557252000 \\
\hline & -9.413925000 & 0.611264000 & -0.038097000 \\
\hline & -10.288401000 & -0.216847000 & -0.090840000 \\
\hline & -9.616362000 & 1.798989000 & 0.016659000 \\
\hline
\end{tabular}

Coordinates of the geometry of isomer ctc of NBT optimized with the M06HF functional

$\begin{array}{lccc}\mathrm{S} & -4.367597000 & -1.309415000 & -0.096739000 \\ \mathrm{~F} & 0.475305000 & 1.512173000 & 1.145863000 \\ \mathrm{~F} & 0.394154000 & 1.579466000 & -1.148613000 \\ \mathrm{~N} & -3.996219000 & 1.250254000 & 0.062550000 \\ \mathrm{~N} & 1.220334000 & -0.442852000 & -0.082989000 \\ \mathrm{~N} & 2.547721000 & 1.670539000 & -0.078537000 \\ \mathrm{~B} & 1.114747000 & 1.087594000 & -0.040630000 \\ \mathrm{C} & -5.767481000 & -0.281966000 & -0.051192000 \\ \mathrm{C} & -7.109291000 & -0.638608000 & -0.091999000 \\ \mathrm{H} & -7.441938000 & -1.662979000 & -0.161258000 \\ \mathrm{C} & -8.010785000 & 0.404637000 & -0.040349000 \\ \mathrm{C} & -7.652715000 & 1.753094000 & 0.048218000\end{array}$




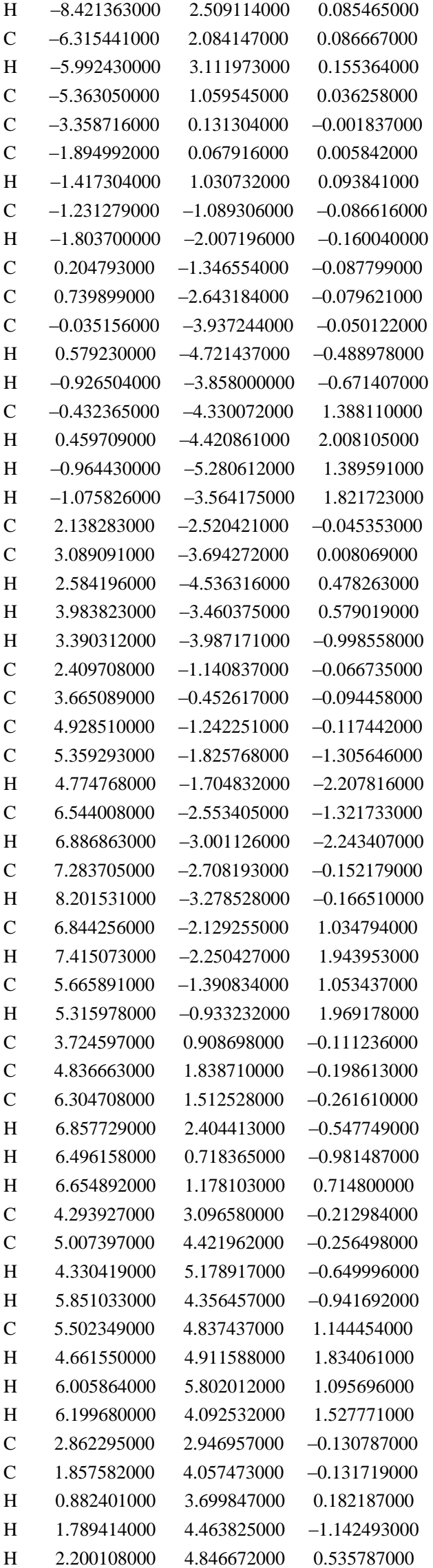


$\begin{array}{cccc}\mathrm{N} & -9.439513000 & 0.069578000 & -0.078507000 \\ \mathrm{O} & -10.235354000 & 0.973969000 & -0.036326000 \\ \mathrm{O} & -9.747828000 & -1.093870000 & -0.150078000\end{array}$

Coordinates of the geometry of isomer ttt of NBT optimized with the M06HF functional

\begin{tabular}{|c|c|c|c|}
\hline & -4.937795000 & -1.928485000 & 0.532455000 \\
\hline & 0.396989000 & 1.485620000 & 1.102949000 \\
\hline & 0.396317000 & 1.645607000 & -1.182107000 \\
\hline$N$ & -3.819059000 & 0.242496000 & -0.320719000 \\
\hline$N$ & 1.256737000 & -0.387913000 & -0.166099000 \\
\hline & 2.522370000 & 1.745415000 & -0.040325000 \\
\hline & 1.100412000 & 1.135846000 & -0.069332000 \\
\hline & -5.962345000 & -0.578546000 & 0.163987000 \\
\hline $\mathrm{C}$ & -7.345270000 & -0.478475000 & 0.259802000 \\
\hline & -7.968163000 & -1.293233000 & 0.595862000 \\
\hline & -7.887385000 & 0.736408000 & -0.102400000 \\
\hline C & -7.139847000 & 1.832710000 & -0.546344000 \\
\hline $\mathrm{H}$ & -7.642132000 & 2.750026000 & -0.809562000 \\
\hline $\mathrm{C}$ & -5.771152000 & 1.711768000 & -0.637004000 \\
\hline $\mathrm{H}$ & -5.156459000 & 2.532307000 & -0.974950000 \\
\hline 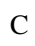 & -5.172558000 & 0.496380000 & -0.279508000 \\
\hline $\mathrm{C}$ & -3.552857000 & -0.956984000 & 0.068402000 \\
\hline$C$ & -2.209380000 & -1.544690000 & 0.150094000 \\
\hline H & -2.144371000 & -2.557892000 & 0.515729000 \\
\hline $\mathrm{C}$ & -1.139203000 & -0.819940000 & 566000 \\
\hline $\mathrm{H}$ & -1.293844000 & 0.204897000 & -0.505607000 \\
\hline F & 0.243740000 & -1.288384000 & -0.169905000 \\
\hline$c$ & 0.780456000 & -2.587073000 & -0.184996000 \\
\hline 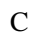 & 0.023817000 & -3.890276000 & -0.2 \\
\hline $\mathrm{H}$ & 0.598625000 & -4.611356000 & -0.804402000 \\
\hline $\mathrm{H}$ & -0.919145000 & -3.746693000 & -0.750605000 \\
\hline $\mathrm{C}$ & -0.226990000 & -4.457667000 & 1.188650000 \\
\hline 11 & 0.724049000 & -4.665496000 & 1.678377000 \\
\hline $\mathrm{H}$ & -0.802498000 & -5.381174000 & 1.131603000 \\
\hline $\mathrm{H}$ & -0.768742000 & -3.739527000 & 1.804138000 \\
\hline $\mathrm{C}$ & 2.179045000 & -2.453248000 & -0.169707000 \\
\hline$C$ & 3.149555000 & -3.611219000 & -0.180192000 \\
\hline 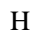 & 2.627423000 & -4.518388000 & 0.115990000 \\
\hline $\mathrm{H}$ & 3.975143000 & -3.441184000 & 0.508016000 \\
\hline $\boldsymbol{H}$ & 3.562502000 & -3.756235000 & -1.178800000 \\
\hline 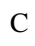 & 2.448627000 & -1.070518000 & -0.159512000 \\
\hline $\mathrm{C}$ & 3.686923000 & -0.359706000 & -0.131611000 \\
\hline $\mathrm{C}$ & 4.964236000 & -1.126542000 & -0.156295000 \\
\hline 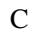 & 5.430100000 & -1.657179000 & -1.356249000 \\
\hline & 4.863264000 & -1.509652000 & -2.265714000 \\
\hline $\mathrm{C}$ & 6.627216000 & -2.364054000 & -1.374390000 \\
\hline $\mathrm{H}$ & 6.997530000 & -2.770019000 & -2.305107000 \\
\hline $\mathrm{C}$ & 7.344732000 & -2.551081000 & -0.195627000 \\
\hline & 8.272345000 & -3.105067000 & -0.211714000 \\
\hline $\mathrm{C}$ & 6.869896000 & -2.026045000 & 1.002987000 \\
\hline $\mathrm{H}$ & 7.423584000 & -2.173133000 & 1.918865000 \\
\hline 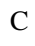 & 5.678669000 & -1.308609000 & 1.024237000 \\
\hline 1 & 5.301340000 & -0.892088000 & 1.948975000 \\
\hline C & 3.714828000 & 1.005036000 & -0.082843000 \\
\hline & 4.809131000 & 1.956386000 & -0.104681000 \\
\hline
\end{tabular}




$\begin{array}{cccc}\mathrm{C} & 6.284280000 & 1.658472000 & -0.146781000 \\ \mathrm{H} & 6.826113000 & 2.564186000 & -0.409473000 \\ \mathrm{H} & 6.503584000 & 0.878043000 & -0.873516000 \\ \mathrm{H} & 6.622903000 & 1.316410000 & 0.831215000 \\ \mathrm{C} & 4.243954000 & 3.205883000 & -0.077132000 \\ \mathrm{C} & 4.934157000 & 4.543930000 & -0.047280000 \\ \mathrm{H} & 4.259348000 & 5.302862000 & -0.440878000 \\ \mathrm{H} & 5.805406000 & 4.517385000 & -0.699991000 \\ \mathrm{C} & 5.366708000 & 4.917400000 & 1.385762000 \\ \mathrm{H} & 4.497766000 & 4.953252000 & 2.043145000 \\ \mathrm{H} & 5.855391000 & 5.890665000 & 1.392050000 \\ \mathrm{H} & 6.060660000 & 4.169742000 & 1.769893000 \\ \mathrm{C} & 2.816569000 & 3.028917000 & -0.031708000 \\ \mathrm{C} & 1.788708000 & 4.118124000 & -0.010128000 \\ \mathrm{H} & 0.820112000 & 3.730626000 & 0.289416000 \\ \mathrm{H} & 1.715153000 & 4.547887000 & -1.010855000 \\ \mathrm{H} & 2.109497000 & 4.897694000 & 0.678843000 \\ \mathrm{~N} & -9.345038000 & 0.882739000 & -0.016653000 \\ \mathrm{O} & -9.825354000 & 1.950449000 & -0.304526000 \\ \mathrm{O} & -9.99198900 & -0.071146000 & 0.337182000\end{array}$

Coordinates of the geometry of isomer ttc of NBT optimized with the M06HF functional

$\begin{array}{cccc}\text { S } & -3.947283000 & 0.518655000 & -0.486232000 \\ \mathrm{~F} & 0.341746000 & 1.452796000 & 1.071332000 \\ \mathrm{~F} & 0.371107000 & 1.599109000 & -1.212942000 \\ \mathrm{~N} & -4.581885000 & -1.798904000 & 0.481301000 \\ \mathrm{~N} & 1.247701000 & -0.414716000 & -0.174219000 \\ \mathrm{~N} & 2.481537000 & 1.735645000 & -0.042771000 \\ \mathrm{~B} & 1.069486000 & 1.106777000 & -0.087443000 \\ \mathrm{C} & -5.623543000 & 0.200532000 & -0.163826000 \\ \mathrm{C} & -6.718673000 & 1.034570000 & -0.351844000 \\ \mathrm{H} & -6.630462000 & 2.035657000 & -0.745421000 \\ \mathrm{C} & -7.943648000 & 0.505790000 & -0.001966000 \\ \mathrm{C} & -8.130189000 & -0.781709000 & 0.510661000 \\ \mathrm{H} & -9.123612000 & -1.119926000 & 0.759199000 \\ \mathrm{C} & -7.029830000 & -1.592240000 & 0.688723000 \\ \mathrm{H} & -7.128308000 & -2.592438000 & 1.082798000 \\ \mathrm{C} & -5.764767000 & -1.098419000 & 0.350112000 \\ \mathrm{C} & -3.572097000 & -1.097271000 & 0.095769000 \\ \mathrm{C} & -2.199361000 & -1.607180000 & 0.152974000 \\ \mathrm{H} & -2.120963000 & -2.610691000 & 0.542996000 \\ \mathrm{C} & -1.141996000 & -0.876923000 & -0.211451000 \\ \mathrm{H} & -1.283610000 & 0.142429000 & -0.553337000 \\ \mathrm{C} & 0.247323000 & -1.328503000 & -0.182813000 \\ \mathrm{C} & 0.799518000 & -2.619787000 & -0.192350000 \\ \mathrm{C} & 0.059855000 & -3.932642000 & -0.235611000 \\ \mathrm{H} & 0.640401000 & -4.641875000 & -0.823986000 \\ \mathrm{H} & -0.889026000 & -3.797790000 & -0.752864000 \\ \mathrm{C} & -0.174321000 & -4.513096000 & 1.175616000 \\ \mathrm{H} & 0.782103000 & -4.706999000 & 1.660697000 \\ \mathrm{H} & -0.733421000 & -5.446256000 & 1.114347000 \\ \mathrm{H} & -0.727327000 & -3.808975000 & 1.797273000 \\ \mathrm{C} & 2.196765000 & -2.467417000 & -0.169753000 \\ \mathrm{C} & 3.181895000 & -3.612853000 & -0.176934000 \\ \mathrm{H} & 2.668929000 & -4.527608000 & 0.111517000\end{array}$




$\begin{array}{cccc}\mathrm{H} & 4.000281000 & -3.435252000 & 0.517984000 \\ \mathrm{H} & 3.603875000 & -3.748615000 & -1.173088000 \\ \mathrm{C} & 2.448351000 & -1.081788000 & -0.159201000 \\ \mathrm{C} & 3.676505000 & -0.353564000 & -0.122376000 \\ \mathrm{C} & 4.963945000 & -1.103497000 & -0.139356000 \\ \mathrm{C} & 5.444894000 & -1.622776000 & -1.338471000 \\ \mathrm{H} & 4.882806000 & -1.477713000 & -2.251253000 \\ \mathrm{C} & 6.650155000 & -2.315620000 & -1.351273000 \\ \mathrm{H} & 7.031721000 & -2.713049000 & -2.281179000 \\ \mathrm{C} & 7.361319000 & -2.499754000 & -0.168179000 \\ \mathrm{H} & 8.295181000 & -3.043105000 & -0.180006000 \\ \mathrm{C} & 6.871543000 & -1.985976000 & 1.029392000 \\ \mathrm{H} & 7.420458000 & -2.130793000 & 1.948493000 \\ \mathrm{C} & 5.671903000 & -1.282550000 & 1.045515000 \\ \mathrm{H} & 5.283133000 & -0.875039000 & 1.969573000 \\ \mathrm{C} & 3.684585000 & 1.011375000 & -0.073371000 \\ \mathrm{C} & 4.765827000 & 1.977919000 & -0.085495000 \\ \mathrm{C} & 6.245030000 & 1.698772000 & -0.110969000 \\ \mathrm{H} & 6.778492000 & 2.609905000 & -0.371579000 \\ \mathrm{H} & 6.481808000 & 0.917716000 & -0.831487000 \\ \mathrm{H} & 6.577095000 & 1.364918000 & 0.872126000 \\ \mathrm{C} & 4.183449000 & 3.219643000 & -0.064758000 \\ \mathrm{C} & 4.853508000 & 4.567700000 & -0.028893000 \\ \mathrm{H} & 4.174871000 & 5.314853000 & -0.438454000 \\ \mathrm{H} & 5.737062000 & 4.550913000 & -0.665186000 \\ \mathrm{C} & 5.253743000 & 4.954641000 & 1.410085000 \\ \mathrm{H} & 4.372411000 & 4.979742000 & 2.051395000 \\ \mathrm{H} & 5.726468000 & 5.935717000 & 1.420364000 \\ \mathrm{H} & 5.952468000 & 4.219957000 & 1.810352000 \\ \mathrm{C} & 2.758399000 & 3.023170000 & -0.033770000 \\ \mathrm{H} & 1.715389000 & 4.097841000 & -0.026576000 \\ & 0.747538000 & 3.695979000 & 0.255736000 \\ \mathrm{H} & 1.652675000 & 4.528738000 & -1.027553000 \\ \mathrm{O} & -9.1273627000 & 4.880411000 & 0.669042000 \\ \mathrm{H} & -1973769000 & 2.447332000 & -0.669271000\end{array}$


Table S2. Relative electronic energies of the four conformational isomers of BT with a trans configuration for their central [C=C] bond optimized with the restricted B3LYP and M06HF functionals and the corresponding values computed with single-point MP2 calculations $[a]$.

\begin{tabular}{ccccc}
\hline Isomer & $\begin{array}{c}\text { B3LYP } \\
\left(\mathrm{kcal} \mathrm{mol}_{-1}\right)\end{array}$ & $\begin{array}{c}\mathrm{B} 3 \mathrm{LYP} / / \mathrm{MP} 2 \\
(\mathrm{kcal} \mathrm{mol}-1)\end{array}$ & $\begin{array}{c}\text { M06HF } \\
(\mathrm{kcal} \mathrm{mol}-1)\end{array}$ & $\begin{array}{c}\text { M06HF//MP2 } \\
(\mathrm{kcal} \mathrm{mol}-1)\end{array}$ \\
\hline $\boldsymbol{c t t}$ & 1.13 & 5.57 & 1.22 & 2.23 \\
$\boldsymbol{c t c}$ & 1.15 & 1.31 & 1.21 & 2.00 \\
$\boldsymbol{t} t \boldsymbol{t t}$ & 0.36 & 0.66 & 0.39 & 0.77 \\
$t \boldsymbol{t c}$ & 0 & 0 & 0 & 0 \\
\hline
\end{tabular}

[a] Calculations were performed with the $6-311+\mathrm{G}(\mathrm{d}, \mathrm{p})$ basis set and the IEFPCM solvation model for MeCN
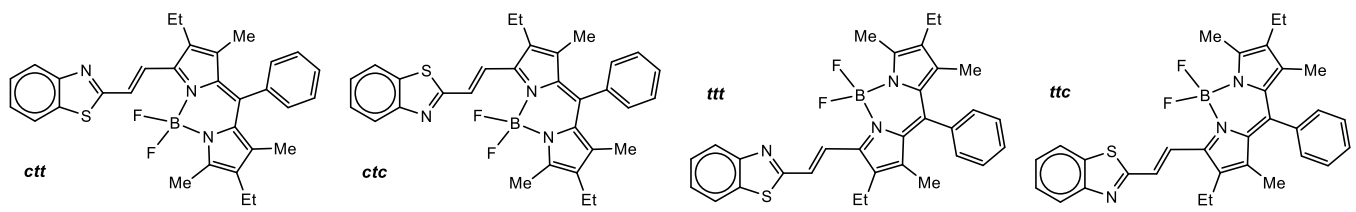

Table S3. Relative electronic energies of the four conformational isomers of NBT with a trans configuration for their central $[\mathrm{C}=\mathrm{C}]$ bond optimized with the restricted B3LYP and M06HF functionals and the corresponding values computed with singlepoint MP2 calculations $[a]$.

\begin{tabular}{ccccc}
\hline Isomer & $\begin{array}{c}\text { B3LYP } \\
\left(\mathrm{kcal} \mathrm{mol}_{-1}\right)\end{array}$ & $\begin{array}{c}\text { B3LYP//MP2 } \\
\left(\mathrm{kcal} \mathrm{mol}_{-1}\right)\end{array}$ & $\begin{array}{c}\text { M06HF } \\
\left(\mathrm{kcal} \mathrm{mol}_{-1}\right)\end{array}$ & $\begin{array}{c}\text { M06HF//MP2 } \\
(\mathrm{kcal} \mathrm{mol}-1)\end{array}$ \\
\hline $\boldsymbol{c t t}$ & 0.79 & 1.62 & 1.00 & 1.91 \\
$\boldsymbol{c t c}$ & 1.09 & 1.24 & 1.33 & 1.68 \\
$\boldsymbol{t t} t$ & 0.14 & 0.78 & 0.23 & 0.62 \\
$\boldsymbol{t} \boldsymbol{c}$ & 0 & 0 & 0 & 0 \\
\hline
\end{tabular}

[a] Calculations were performed with the $6-311+\mathrm{G}(\mathrm{d}, \mathrm{p})$ basis set and the IEFPCM solvation model for MeCN.

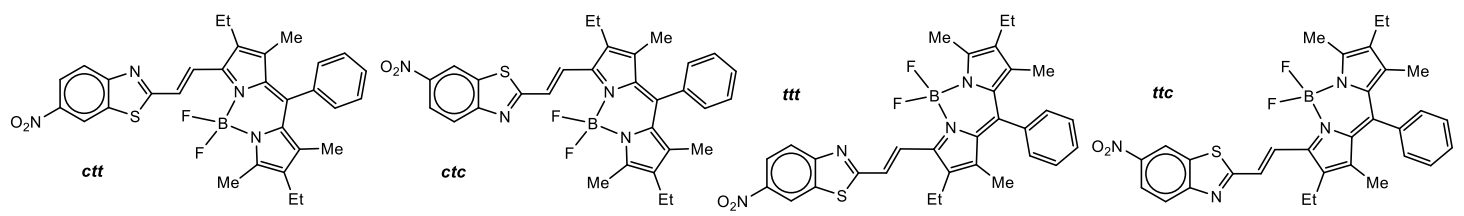


Table S4. Relative free energy $(\Delta G)$ of the four conformational isomers of BT with a trans configuration for their central [C=C] bond as well as vertical energy $(\Delta E)$, wavelength $(\lambda)$, oscillator strength $(f)$ and main orbital pair with its contribution for electronic transitions from the ground state to the corresponding first singlet excited state computed with the restricted B3LYP and M06HF functionals $[a]$.

\begin{tabular}{|c|c|c|c|c|c|c|c|}
\hline Functional & Isomer & $\begin{array}{c}\Delta G \\
(\text { kcal mol-1) }\end{array}$ & $\begin{array}{c}\Delta E \\
(\mathrm{eV})\end{array}$ & $\begin{array}{c}\lambda \\
(\mathrm{nm})\end{array}$ & $f$ & Main Orbital Pair & $\begin{array}{c}\text { Contribution } \\
(\%)\end{array}$ \\
\hline \multicolumn{8}{|l|}{ B3LYP } \\
\hline & $c t t$ & 1.21 & 2.20 & 564 & 1.0376 & {$[\mathrm{HOMO}] \rightarrow[\mathrm{LUMO}]$} & 100 \\
\hline & $c t c$ & 1.12 & 2.21 & 561 & 1.0177 & {$[\mathrm{HOMO}] \rightarrow[\mathrm{LUMO}]$} & 100 \\
\hline & $t t t$ & 0.36 & 2.27 & 546 & 1.0966 & {$[\mathrm{HOMO}] \rightarrow[\mathrm{LUMO}]$} & 99 \\
\hline & $t t c$ & 0 & 2.29 & 542 & 1.1186 & {$[\mathrm{HOMO}] \rightarrow[\mathrm{LUMO}]$} & 99 \\
\hline \multicolumn{8}{|l|}{ M06HF } \\
\hline & $c t t$ & 3.42 & 2.60 & 476 & 0.7667 & {$[\mathrm{HOMO}] \rightarrow[\mathrm{LUMO}]$} & 91 \\
\hline & $c t c$ & 2.74 & 2.58 & 480 & 0.7936 & {$[\mathrm{HOMO}] \rightarrow[\mathrm{LUMO}]$} & 91 \\
\hline & $t t t$ & 0.69 & 2.64 & 471 & 0.8623 & {$[\mathrm{HOMO}] \rightarrow[\mathrm{LUMO}]$} & 91 \\
\hline & $t t c$ & 0 & 2.65 & 467 & 0.8687 & {$[\mathrm{HOMO}] \rightarrow[\mathrm{LUMO}]$} & 91 \\
\hline $\begin{array}{l}{[a] \text { The therr }} \\
\text { photophysical p } \\
\text { computed with } \\
\text { basis set and the I } \\
\text { model for MeCN a }\end{array}$ & $\begin{array}{l}\text { nic and } \\
\text { is } \quad \text { were } \\
11+\mathrm{G}(\mathrm{d}, \mathrm{p}) \\
\text { solvation }\end{array}$ & & & & & & \\
\hline
\end{tabular}

Table S5. Relative free energy $(\Delta G)$ of the four conformational isomers of NBT with a trans configuration for their central [C=C] bond as well as vertical energy $(\Delta E)$, wavelength $(\lambda)$, oscillator strength $(f)$ and main orbital pair with its contribution for electronic transitions from the ground state to the corresponding first singlet excited state computed with the restricted B3LYP and M06HF functionals $[a]$.

\begin{tabular}{|c|c|c|c|c|c|c|c|}
\hline Functional & Isomer & $\begin{array}{c}\Delta G \\
\left(\mathrm{kcal} \mathrm{mol}_{-1}\right)\end{array}$ & $\begin{array}{c}\Delta E \\
(\mathrm{eV})\end{array}$ & $\begin{array}{c}\lambda \\
(\mathrm{nm})\end{array}$ & $f$ & Main Orbital Pair & $\begin{array}{c}\text { Contribution } \\
(\%)\end{array}$ \\
\hline \multicolumn{8}{|l|}{ B3LYP } \\
\hline & $c t t$ & 0.52 & 2.03 & 601 & 1.1610 & {$[\mathrm{HOMO}] \rightarrow[\mathrm{LUMO}]$} & 100 \\
\hline & $c t c$ & 0.99 & 2.05 & 604 & 1.1348 & {$[\mathrm{HOMO}] \rightarrow[$ LUMO $]$} & 100 \\
\hline & $t t t$ & 0.24 & 2.10 & 591 & 1.1430 & {$[\mathrm{HOMO}] \rightarrow[$ LUMO $]$} & 99 \\
\hline & $t t c$ & 0 & 2.09 & 594 & 1.06 & {$[\mathrm{HOMO}] \rightarrow[$ LUMO $]$} & 99 \\
\hline \multicolumn{8}{|l|}{ M06HF } \\
\hline & $c t t$ & 1.21 & 2.60 & 477 & 0.8297 & {$[\mathrm{HOMO}] \rightarrow[$ LUMO $]$} & 90 \\
\hline & $c t c$ & 2.43 & 2.62 & 473 & 0.8174 & {$[\mathrm{HOMO}] \rightarrow[\mathrm{LUMO}]$} & 89 \\
\hline & $t t t$ & 0 & 2.65 & 467 & 0.8973 & {$[\mathrm{HOMO}] \rightarrow[\mathrm{LUMO}]$} & 89 \\
\hline & $t t c$ & 1.32 & 2.67 & 464 & 0.8904 & {$[\mathrm{HOMO}] \rightarrow[\mathrm{LUMO}]$} & 88 \\
\hline $\begin{array}{l}{[a] \text { The thermody }} \\
\text { and photopt } \\
\text { parameters } \\
\text { computed with } \\
311+\mathrm{G}(\mathrm{d}, \mathrm{p}) \text { bas } \\
\end{array}$ & & & & & & & \\
\hline
\end{tabular}

$\mathrm{MeCN}$ at $25^{\circ} \mathrm{C}$. 


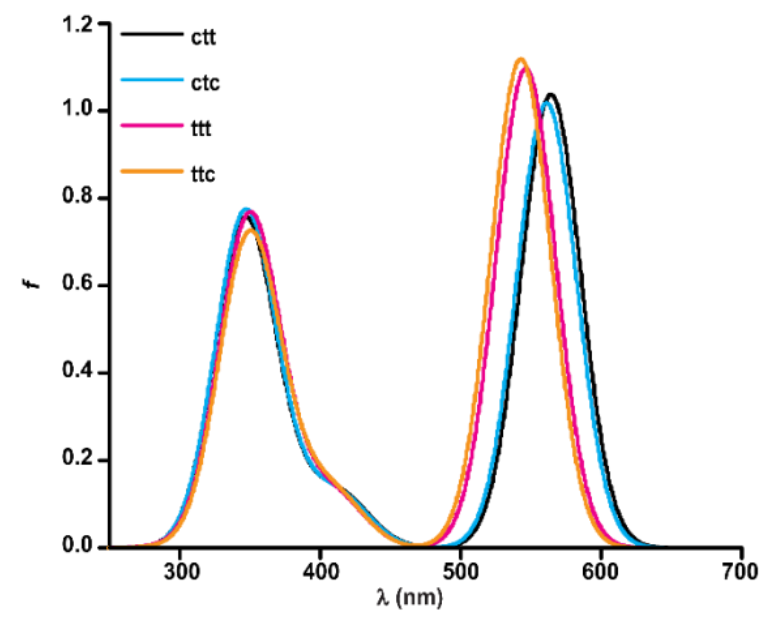

Figure S5. Absorption spectra of the four isomers of BT computed with the restricted B3LYP functional, the 6-311+G(d,p) basis set and the IEFPCM solvation model for MeCN and plotted with Gaussian broadening with a width at half maximum of $50 \mathrm{~nm}$.

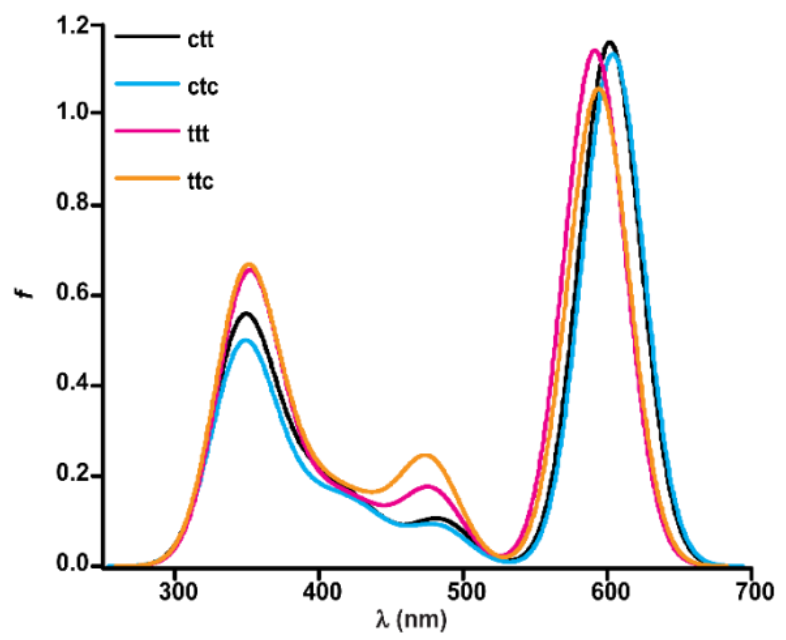

Figure S7. Absorption spectra of the four isomers of NBT computed with the restricted B3LYP functional, the $6-311+\mathrm{G}(\mathrm{d}, \mathrm{p})$ basis set and the IEFPCM solvation model for MeCN and plotted with Gaussian broadening with a width at half maximum of $50 \mathrm{~nm}$.

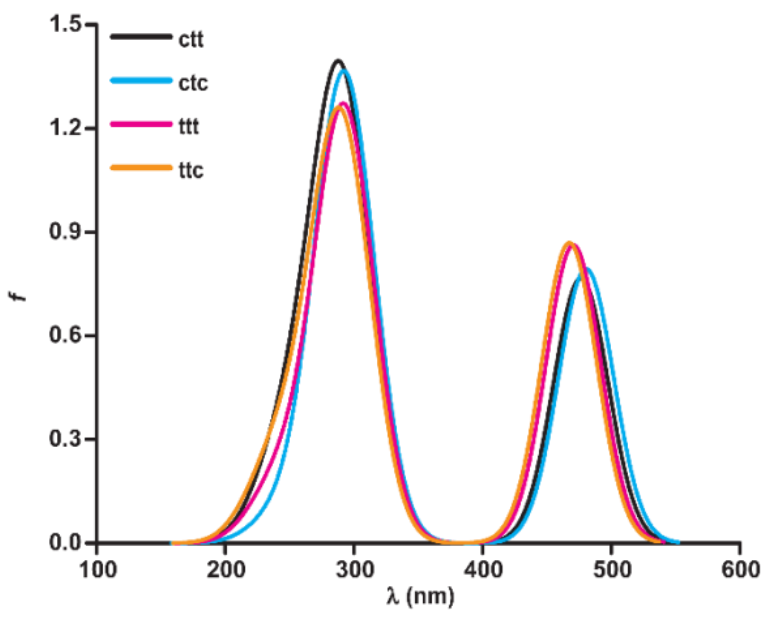

Figure S6. Absorption spectra of the four isomers of BT computed with the restricted M06HF functional, the 6-311+G(d,p) basis set and the IEFPCM solvation model for MeCN and plotted with Gaussian broadening with a width at half maximum of $50 \mathrm{~nm}$.

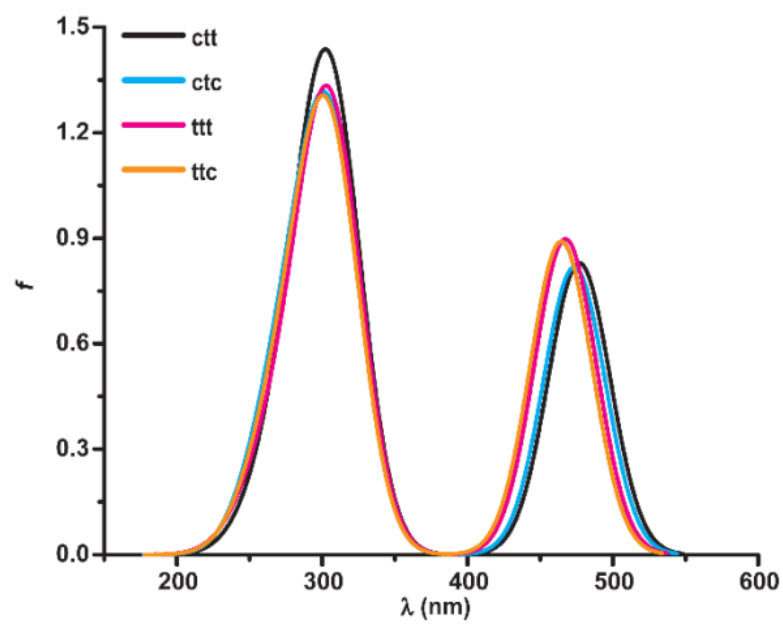

Figure S8. Absorption spectra of the four isomers of NBT computed with the restricted M06HF functional, the 6-311+G(d,p) basis set and the IEFPCM solvation model for MeCN and plotted with Gaussian broadening with a width at half maximum of $50 \mathrm{~nm}$. 
Table S6. Absorption $\left(\lambda_{\mathrm{Ab}}\right)$ and emission $\left(\lambda_{\mathrm{Em}}\right)$ wavelengths, fluorescence quantum yield $(\phi)$, molar absorption coefficient $(\varepsilon)$ and brightness $(\phi \times \varepsilon)$ of BO, BT and NBT in MeCN at $25^{\circ} \mathrm{C}[a]$.

\begin{tabular}{cccccc}
\hline & $\begin{array}{c}\lambda \mathrm{Ab} \\
(\mathrm{nm})\end{array}$ & $\begin{array}{c}\lambda \mathrm{Em} \\
(\mathrm{nm})\end{array}$ & $\phi$ & $\begin{array}{c}\varepsilon \\
(\mathrm{mM}-1 \mathrm{~cm}-1)\end{array}$ & $\begin{array}{c}\phi \times \varepsilon \\
(\mathrm{mM}-1 \mathrm{~cm}-1)\end{array}$ \\
\hline BO & 578 & 593 & 0.83 & 104 & 86 \\
BT & 584 & 597 & 0.85 & 124 & 105 \\
\hline NBT & 592 & 613 & 0.44 & 120 & \\
\hline The values of $\phi$ were determined in aerated solutions against a MeCN solution of $8(\phi=0.50)$ & & & &
\end{tabular}

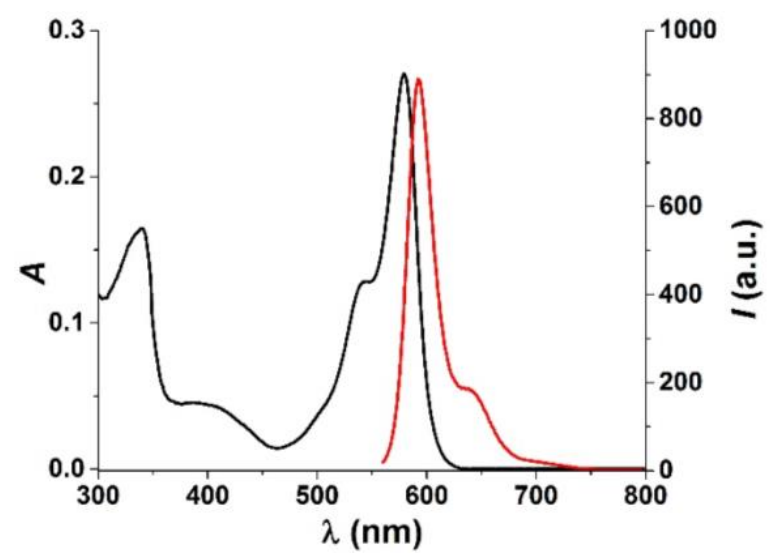

Figure S9. Absorption and emission $\left(\lambda_{\mathrm{Ex}}=550 \mathrm{~nm}\right)$ spectra of $\mathbf{B O}$ $(2.5 \mu \mathrm{M})$ in $\mathrm{MeCN}$ at $25^{\circ} \mathrm{C}$.

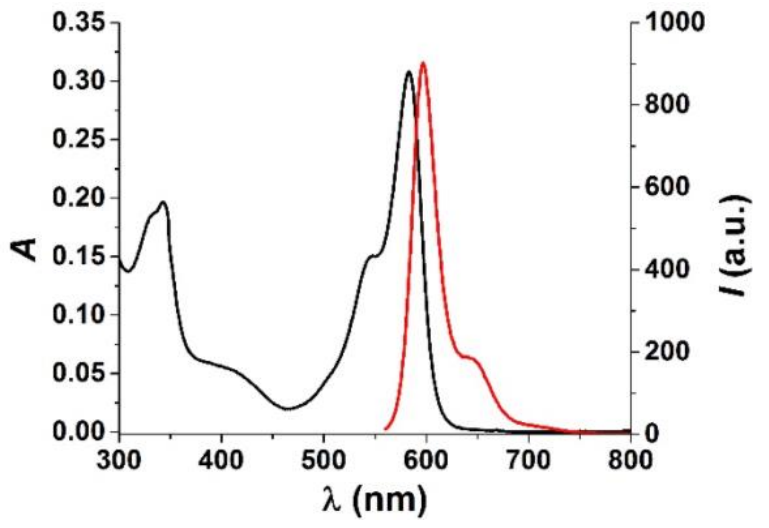

Figure S10. Absorption and emission $\left(\lambda_{\mathrm{Ex}}=550 \mathrm{~nm}\right)$ spectra of $\mathbf{B T}$ $(2.5 \mu \mathrm{M})$ in $\mathrm{MeCN}$ at $25^{\circ} \mathrm{C}$.

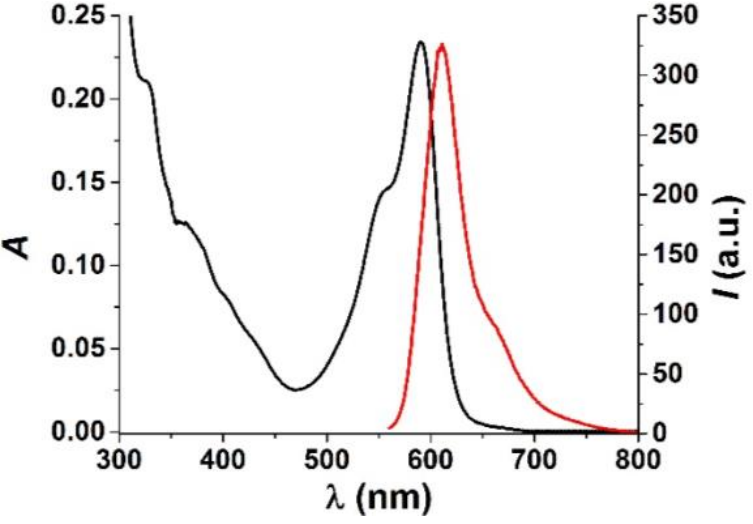

Figure S11. Absorption and emission $\left(\lambda_{\mathrm{Ex}}=560 \mathrm{~nm}\right)$ spectra of NBT $(2 \mu \mathrm{M})$ in $\mathrm{MeCN}$ at $25^{\circ} \mathrm{C}$. 

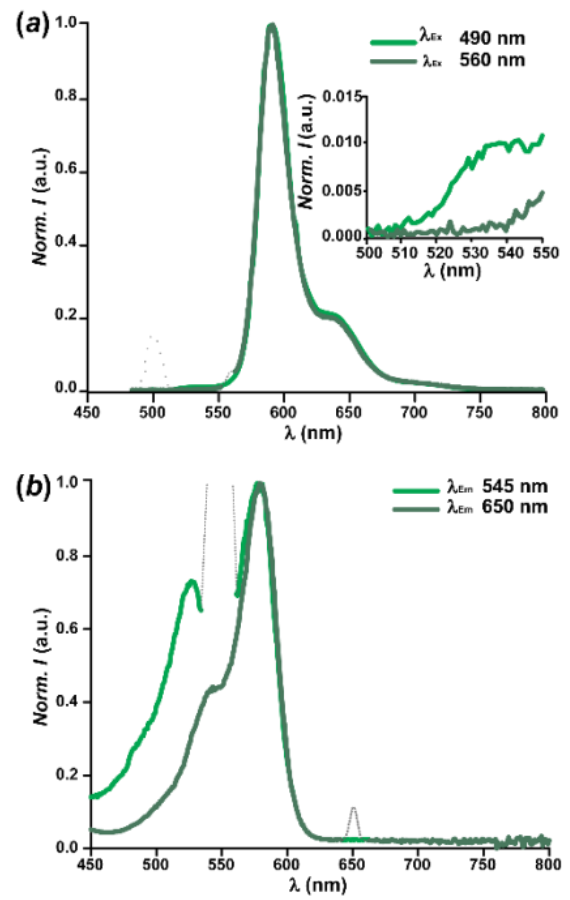

Figure S12. Normalized emission (A) and excitation (B) spectra of

BO in $\mathrm{MeCN}$ at $25^{\circ} \mathrm{C}$.
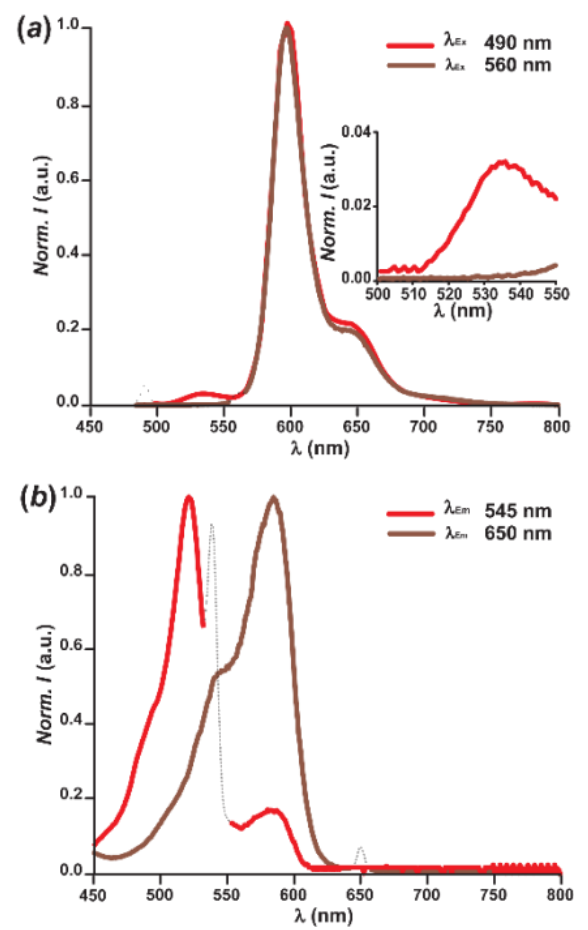
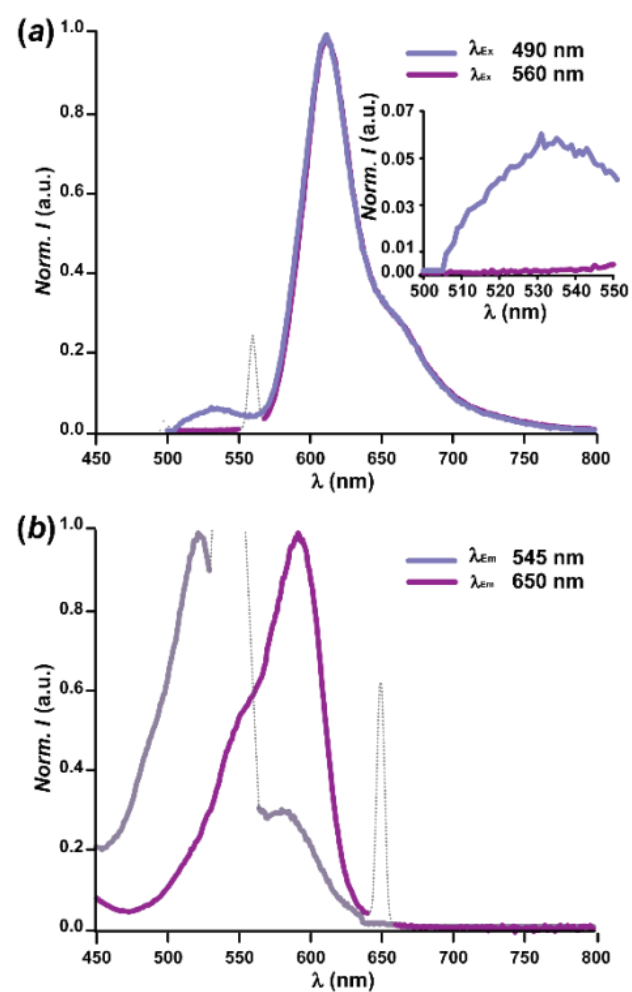

Figure S13. Normalized emission (A) and excitation (B) spectra of BT in $\mathrm{MeCN}$ at $25^{\circ} \mathrm{C}$.
Figure S14. Normalized emission (A) and excitation (B) spectra of NBT in $\mathrm{MeCN}$ at $25^{\circ} \mathrm{C}$. 

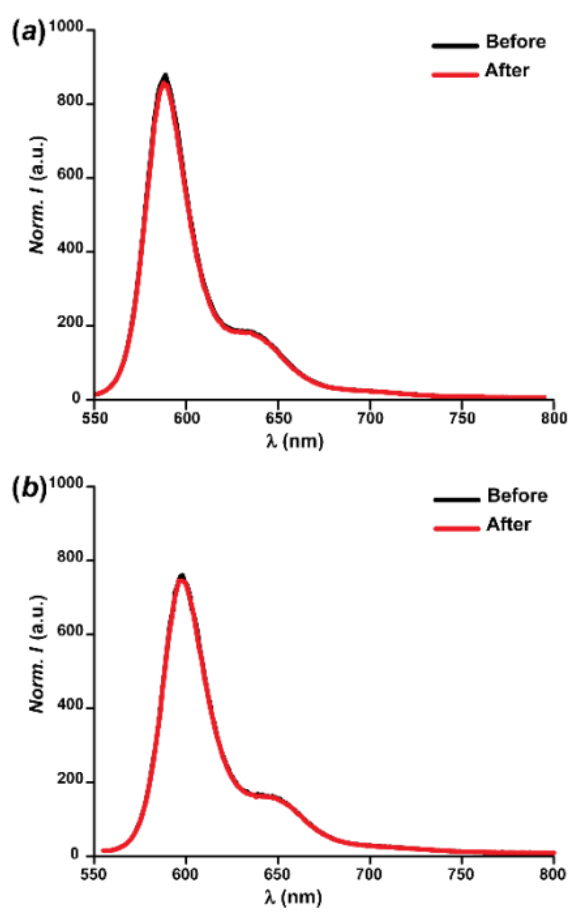

Figure S15. Emission spectra $\left(\lambda_{\mathrm{Ex}}=550 \mathrm{~nm}\right)$ of $\mathbf{B O}$ in $\mathrm{MeCN}(6$ $\mu \mathrm{M})$ recorded before and after irradiation at either (A) $300-410 \mathrm{~nm}$ $(3.70 \mathrm{~mW} \mathrm{~cm}-2)$ or $($ B) $500-530 \mathrm{~nm}(0.18 \mathrm{~mW} \mathrm{~cm}-2)$ for $240 \mathrm{~s}$ at $25{ }^{\circ} \mathrm{C}$.
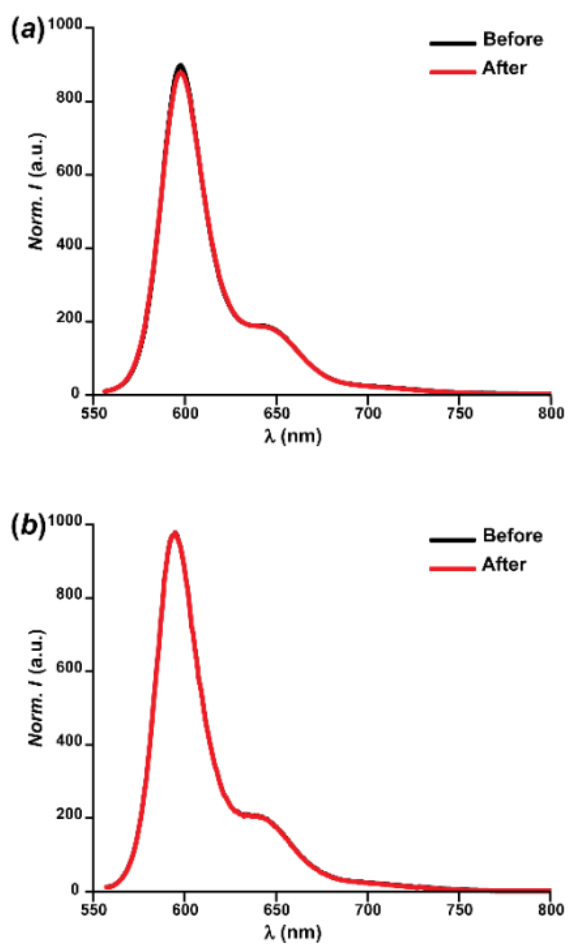

Figure S16. Emission spectra $\left(\lambda_{\mathrm{Ex}}=550 \mathrm{~nm}\right)$ of BT in $\mathrm{MeCN}(5 \mu \mathrm{M})$ recorded before and after irradiation at either (A) 300-410 nm (3.70 $\mathrm{mW} \mathrm{cm}-2)$ or (B) $500-530 \mathrm{~nm}(0.18 \mathrm{~mW} \mathrm{cm-2})$ for $240 \mathrm{~s}$ at $25^{\circ} \mathrm{C}$.
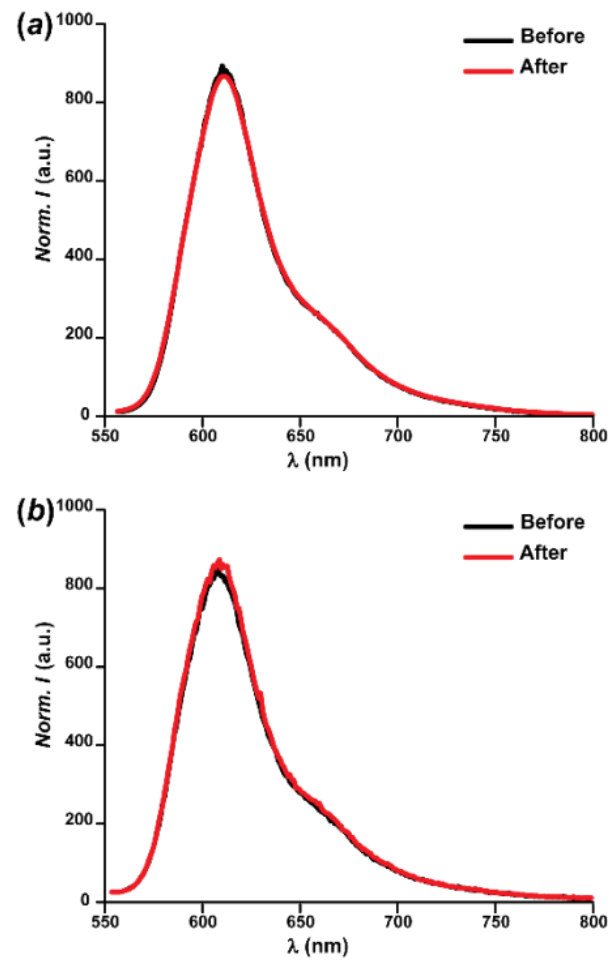

Figure S17. Emission spectra $\left(\lambda_{\mathrm{Ex}}=550 \mathrm{~nm}\right)$ of NBT in $\mathrm{MeCN}$ ( 6 or $3 \mu \mathrm{M}$ ) recorded before and after irradiation at either (A) $300-410 \mathrm{~nm}$ (3.70 mW cm-2) or (B) $500-530 \mathrm{~nm}(0.18 \mathrm{~mW} \mathrm{cm-2)} \mathrm{for} 240 \mathrm{~s}$ at 25 ${ }^{\circ} \mathrm{C}$ 

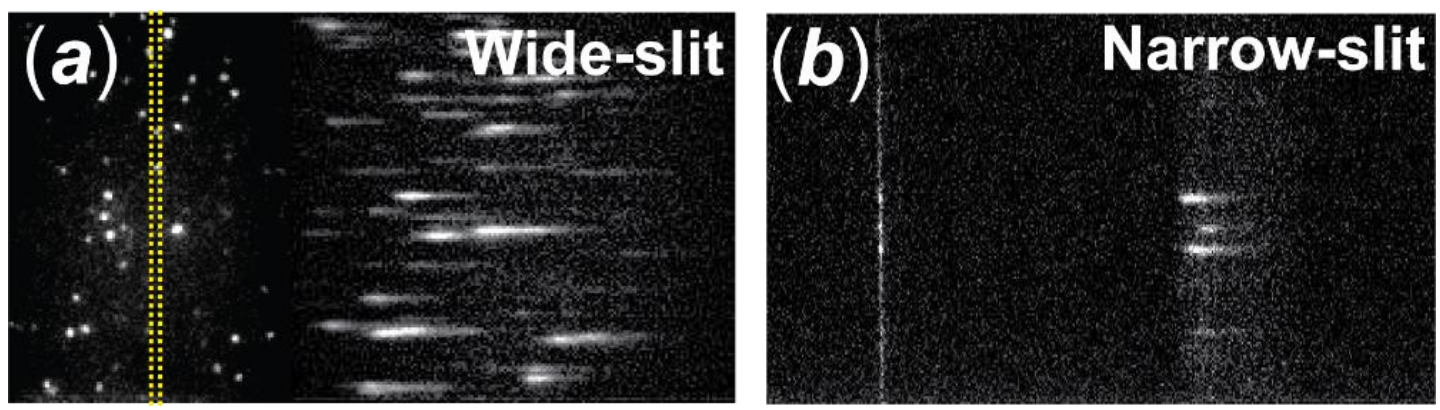

Figure S18. Raw images of $(\boldsymbol{a})$ wide-slit and $(\boldsymbol{b})$ narrow-slit configurations with $5 \mathrm{~mm}$ and $50 \mu \mathrm{m}$ entrance slit widths respectively. Yellow dashed box in $(\boldsymbol{a})$ indicates the adjustment of entrance slit width and the confinement of FOV to minimize the detection of multiple single molecules with overlapping spectra along similar horizontal coordinate.
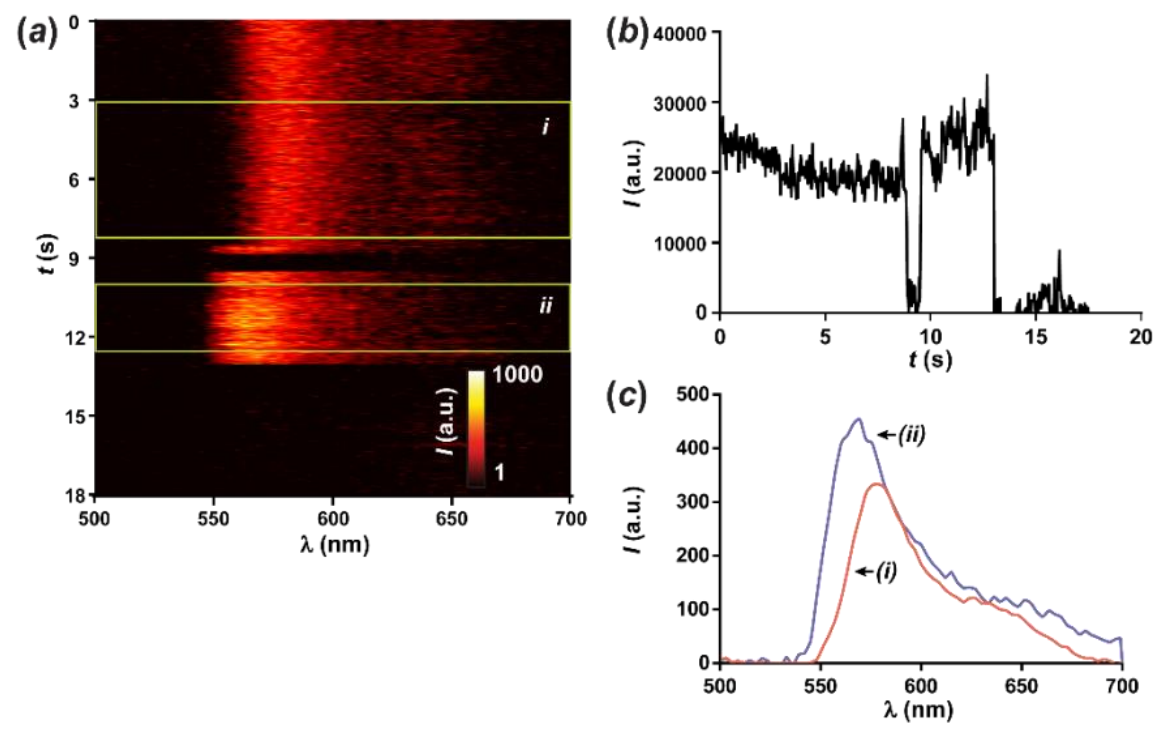

Figure S19. Emission spectra $(\boldsymbol{a})$ and intensity evolutions $(\boldsymbol{b})$ of a single BO molecule together with the averaged emission spectra $(\boldsymbol{c})$ from 3.2-8.8 s $(\boldsymbol{i})$ and $10.3-12.4 \mathrm{~s}(\boldsymbol{i i})$ of the acquisition duration. 

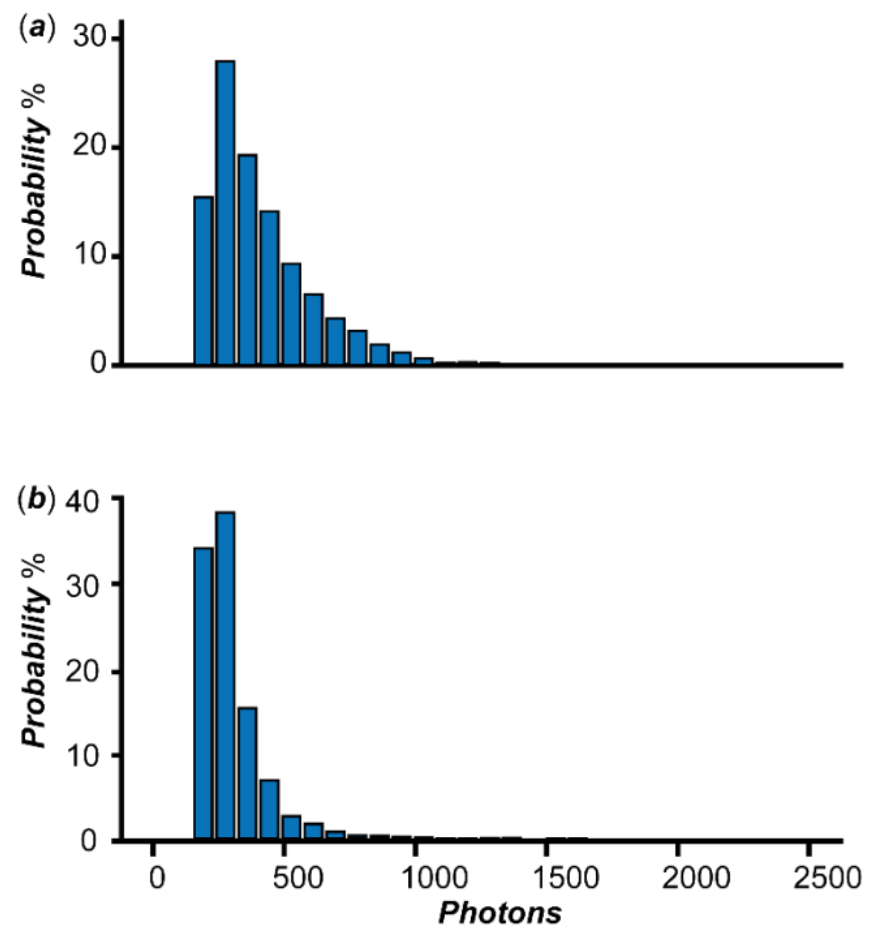

Figure S20. Histograms of photon distributions of $\mathbf{B O}$ single molecules in the spectral window of 550-600 nm (a) and 600-650 nm (b).
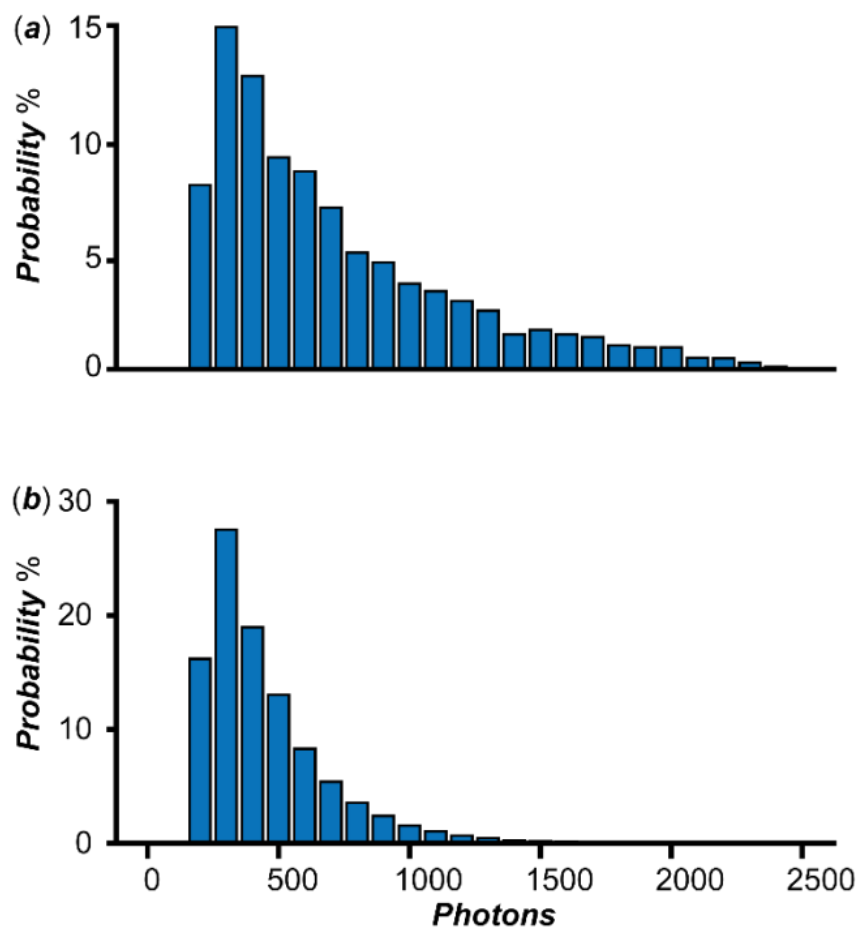

Figure S21. Histograms of photon distributions of BT single molecules in the spectral window of 550-600 nm $(\boldsymbol{a})$ and $600-650 \mathrm{~nm}(\boldsymbol{b})$.
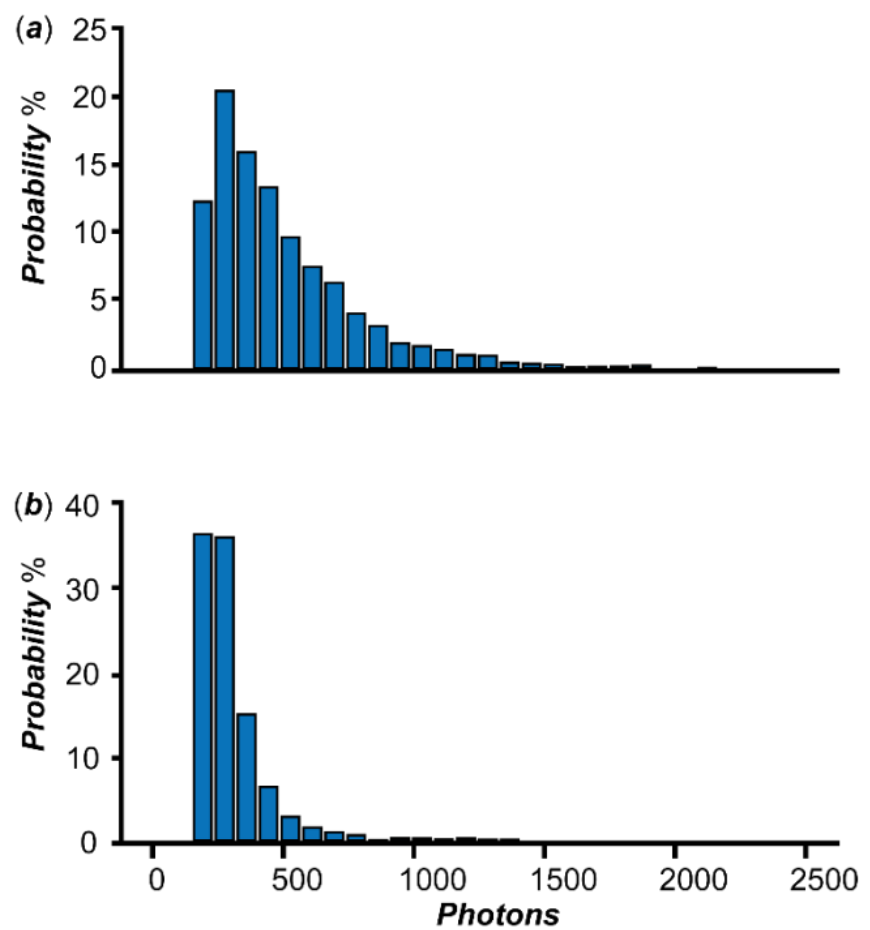

Figure S22. Histograms of photon distributions of NBT single molecules in the spectral window of 550-600 $\mathrm{nm}(\boldsymbol{a})$ and $600-650 \mathrm{~nm}(\boldsymbol{b})$. 

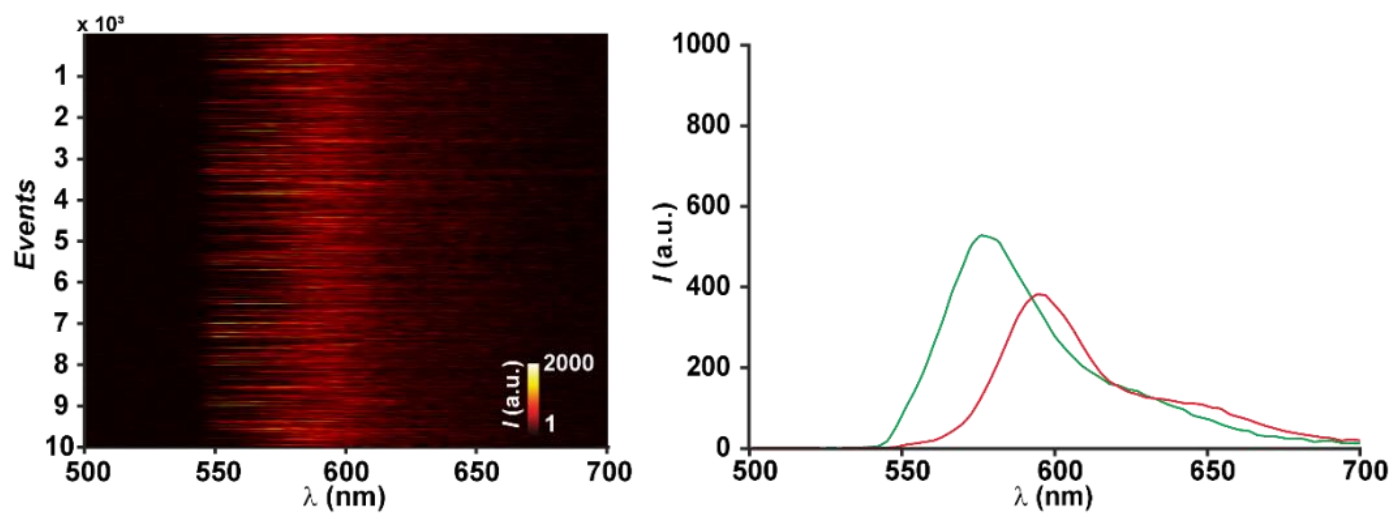

Figure S23. 10,052 single-molecule emission spectra of $\mathbf{B O}$ and the averaged emission spectra extracted from the molecules with spectral centroid of 590-596 nm (green line) and 611-617 nm (red line).
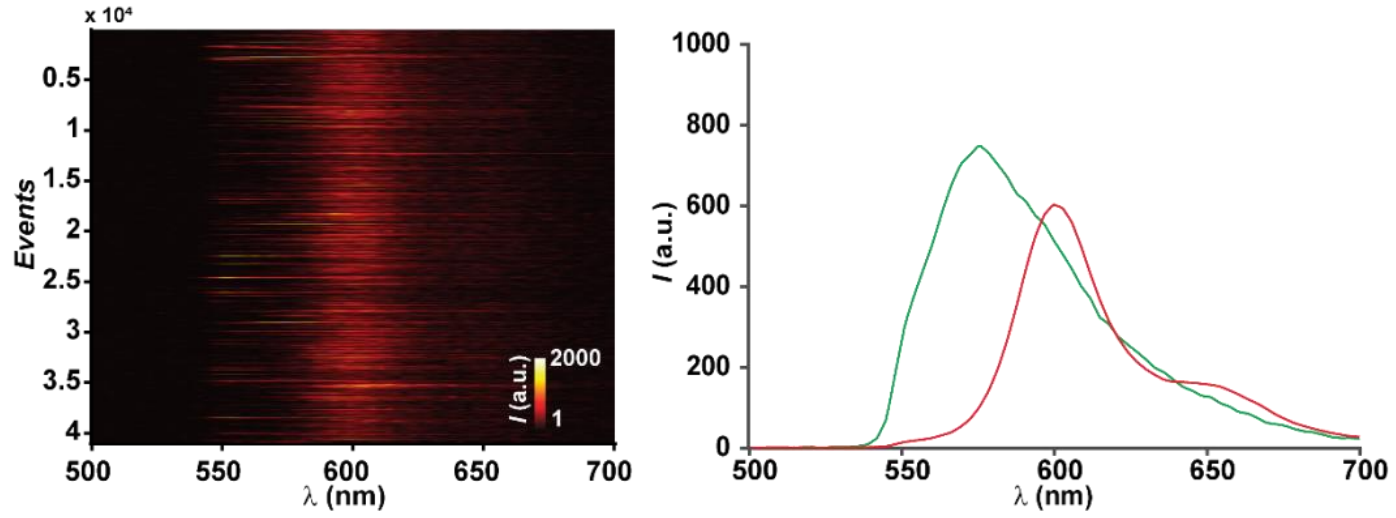

Figure S24. 40,949 single-molecule emission spectra of BT and the averaged emission spectra extracted from the molecules with spectral centroid of 590-596 nm (green line) and 611-617 nm (red line).
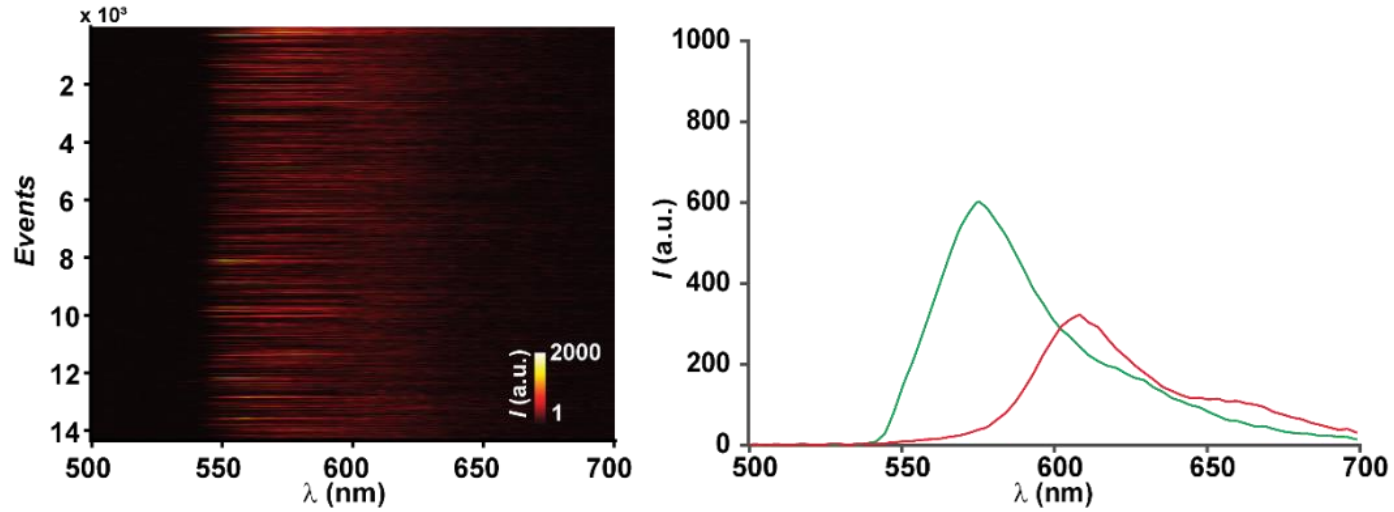

Figure S25. 14,349 single-molecule emission spectra of NBT and the averaged emission spectra extracted from the molecules with spectral centroid of 590-596 nm (green line) and 620-626 nm (red line). 